\title{
NANOINDENTATION TECHNIQUES FOR THE EVALUATION OF SILICON NITRIDE THIN FILMS
}

\author{
A Thesis \\ presented to \\ the Faculty of California Polytechnic State University, \\ San Luis Obispo \\ In Partial Fulfillment \\ of the Requirements for the Degree \\ Master of Science in Biomedical Engineering
}

by

Weston Thomas Mangin

December 2016 
(C) 2016

Weston Thomas Mangin

\section{ALL RIGHTS RESERVED}




\section{COMMITTEE MEMBERSHIP}

TITLE: Nanoindentation Techniques for the Evaluation of

Silicon Nitride Thin Films

AUTHOR: $\quad$ Weston Thomas Mangin

DATE SUBMITTED: December 2016

COMMITTEE CHAIR: $\quad$ Lanny Griffin, Ph.D.

Professor of Biomedical Engineering

COMMITEE MEMBER: Dan Walsh, Ph.D.

Professor of Biomedical Engineering

COMMITTEE MEMBER: $\quad$ Satinderpall S. Pannu, Ph.D.

Section Leader for Micro and Nano Technology,

Lawrence Livermore National Laboratory 


\begin{abstract}
Nanoindentation Techniques for the Evaluation of Silicon Nitride Thin Films Weston Thomas Mangin
\end{abstract}

Silicon nitride thin films are of interest in the biomedical engineering field due to their biocompatibility and favorable tribological properties. Evaluation and understanding of the properties of these films under diverse loading and failure conditions is a necessary prerequisite to their use in biomedical devices. Three wafers of silicon nitride-coated silicon were obtained from Lawrence Livermore National Laboratory and used to create 96 samples. Samples were subjected to nanoindentation testing to evaluate the mechanical properties of the film. Samples were subjected to nanoimpact testing to compare the damage resistance of the film to separate nanoimpact types. Samples were subjected to nanoscratch testing to evaluate the consistency of the critical load of the film. Results showed that there were no significant differences in the mechanical properties of the film across the tested groups. There was a significant difference observed in the rate of damage to the film between pendulum oscillation nanoimpact testing and sample oscillation nanoimpact testing, with the former causing more damage with all experiment variables controlled for. Results showed that the critical load measure for the film was significantly different between different nanoscratch test parameters. The conclusions from this study will support future work for in vitro and in vivo testing of ceramic thin films for biomedical applications.

Keywords: Nanoindentation, Nanoimpact, Nanoscratch, Silicon Nitride 


\section{ACKNOWLEDGMENTS}

Thank you to Dr. Satinderpall Pannu, who supported this process at the beginning by providing my sample materials, and at the end by agreeing to sit on my committee and review the work that had been done.

Thank you to Dr. Dan Walsh, who helped to refine an interest in these materials and agreed to review my work even when it was not convenient for him.

Thank you to Dale and Sandy Arnold, whose support made finishing this much easier than it could have been.

Thank you to Dr. Lanny Griffin, whose patience, confidence, and guidance made this project possible. Without his continued willingness to guide me forward, this thesis would not exist.

And thank you to my parents, whose patient and loving support is the only reason that I am even in a position to be typing out this page. You have done everything that you could to develop an nurture me, and I hope that this serves as a tangible result of that effort. 


\section{TABLE OF CONTENTS}

Page

LIST OF TABLES

viii

LIST OF FIGURES

ix

\section{CHAPTER}

1.0 Introduction and Background

1.1 Silicon Nitride and Silicon Nitride Coatings

1.2 Silicon nitride film applications

1.3 Nanoindentation Theory

1.4 Nanoimpact Theory

1.5 Nanoscratch Theory

2.0 Research Objectives

3.0 Materials and Methods

3.1 Materials

3.2 Device and Device Calibrations

3.3 Test types

23

4.0 Results

31

4.1 Microscope Evaluation

4.2 Silicon Nitride Nanoindentation Results

4.2.1 Nanoindentation Data Analysis Rationale

4.2.2 Nanoindentation Elastic Modulus Results

4.2.3 Nanoindentation Elastic Modulus Statistical Analysis

4.2.4 Nanoindentation Hardness Results

4.2.5 Nanoindentation Hardness Statistical Analysis 
4.3 Silicon Nitride Nanoimpact Results

4.3.1 Nanoimpact Data Analysis Rationale

4.3.2 Nanoimpact Statistical Analysis

4.4 Silicon Nitride Nanoscratch Results

4.4.1 Nanoscratch Data Analysis Rationale

4.4.2 Nanoscratch Critical Load Statistical Analysis

5.0 Discussion

6.0 Conclusions and Recommendations 55

REFERENCES 58

APPENDIX: Data Statistics

61 
Table

\section{LIST OF TABLES}

Table 4.1: Parameters used for nanoindentation testing. 36

Table 4.2: Pyramidal analysis parameters for analysis of nanoindentation data. 37

Table 4.3: Analysis function parameters for analysis of nanoindentation data. 37

Table 4.4: Results of the Shapiro-Wilk Test for nanoindentation elastic modulus. 39

Table 4.5: Results of the ANOVA for nanoindentation elastic modulus. 40

Table 4.6: Results of the Shapiro-Wilk Test for nanoindentation hardness. 41

Table 4.7: Results of the ANOVA for nanoindentation hardness. 41

Table 4.8: Sample oscillation test parameters 42

Table 4.9: Pendulum oscillation test parameters 42

Table 4.10: Results of the Shapiro-Wilk Test for nanoimpact sample oscillation results. 43

Table 4.11: Results of the Kolmogorov's D Test for nanoimpact sample oscillation results.

Table 4.12: Results of the Kolmogorov's D Test for nanoimpact pendulum oscillation results.

Table 4.13: Results of the Shapiro-Wilk W test for the transformed sample oscillation data

Table 4.14: Results of the Shapiro-Wilk W test for the transformed pendulum oscillation data

Table 4.15: Results of ANOVA for nanoimpact testing

Table 4.16: Parameters used in nanoscratch testing.

Table 4.17: Results of the Shapiro-Wilk Test for nanoscratch critical load.

Table 4.18: Results of a combined ANOVA for critical load. 
Figure

\section{LIST OF FIGURES}

Figure 1.1: Scanning electron microscopy images of silicon nitride grains (d) showing the acicular grain formation.

Figure 1.2: An example of the fracture path through a material with a more typical grain structure (a) and through the acicular grain structure of silicon nitride (b).

Figure 1.3: An idealized nanoindentation loading-unloading curve.

Figure 3.1: The crystallographic planes of a $<110>$ orientation silicon wafer.

Figure 3.2: A Berkovich indenter tip in the NanoTest module. The pendulum is to the left of the indenter tip.

Figure 3.3: The selection of indenter tips used in this study. From left to right: Berkovich, Cube Corner, and Conical.

Figure 3.4: A typical nanoindentation test result, displaying a depth and load curve.

Figure 3.5: A failed nanoindentation. Note the random and meaningless depth measurements.

Figure 3.6: A typical nanoimpact result, with the measured depth increasing on each impulse.

Figure 3.7: A failed nanoimpact result.

Figure 3.8: A typical nanoscratch result. The upper line is the load, while the lower line is the topographical trace.

Figure 4.1: A typical nanoscratch result. The upper line is the load, while the lower line is the topographical trace.

Figure 4.2: An example of a nanoindentation test. The row of indents runs diagonally from the upper left to the lower right and appears as green triangles against the purple nitride background.

Figure 4.3: Impulse tests carried out for calibration in the same format as the data tests. The impulse sites appear as irregular green dots in diagonal rows from the upper left to the lower right. 
Figure 4.4: An example of a nanoscratch image. There are three vertical scratches in this image, parallel to each other and near the center of the field. They appear as red-orange discolorations, just above each of the black label lines.

Figure 4.5: Test type effect on the rate of change of depth for silicon nitride thin films.

Figure 4.6: The effect of the Quadrant (Test) on the critical load.

Figure 4.7: The effect of the wafer on the critical load.

Figure 6.1: An example of a nanoimpact test site with a significant debris field. Note that the shape of the cube corner indenter is distorted. 


\subsection{Introduction and Background}

\subsection{Silicon Nitride and Silicon Nitride Coatings}

Silicon nitride is a ceramic material with the formula $\mathrm{Si}_{3} \mathrm{~N}_{4}$. In most applications, it is used for either its very high resistance to wear, ability to hold up under very high temperatures, or both. Bulk silicon nitride is generally reported as having a Young's modulus near $300 \mathrm{GPa}$ and a Vickers hardness of at least $12 \mathrm{GPa}$ [1]. These properties are part of the reason that the compound is highly resistant to wear, but this ceramic has additional advantages. The crystal structure of silicon nitride is very important as well: it tends to form highly acicular grains (Fig. 1.1) [2].

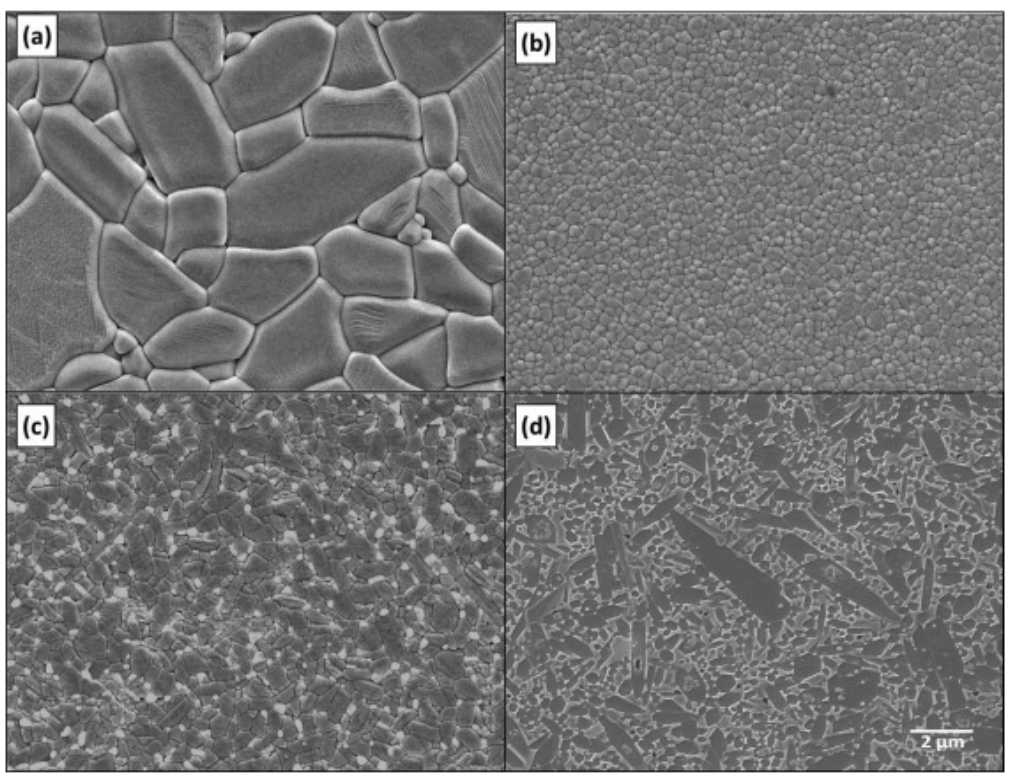

Figure 1.1: Scanning electron microscopy images of

silicon nitride grains (d) showing the acicular grain formation. [1] 
The acicular grain structure offers superior resistance to crack propagation through the silicon nitride film [2]. With additional processing performed on the material, a vitreous phase can form between the grains that toughens it further, allowing for fracture toughness values as high as $10 \mathrm{MPa} \mathrm{m}^{1 / 2}[3]$. When a crack begins to propagate through a silicon nitride structure, the combination of the acicular grains and the vitreous intergrain phase force the crack to take a tortuous path, dissipating far more energy as the crack grows than would be necessary in a typical ceramic material [1].
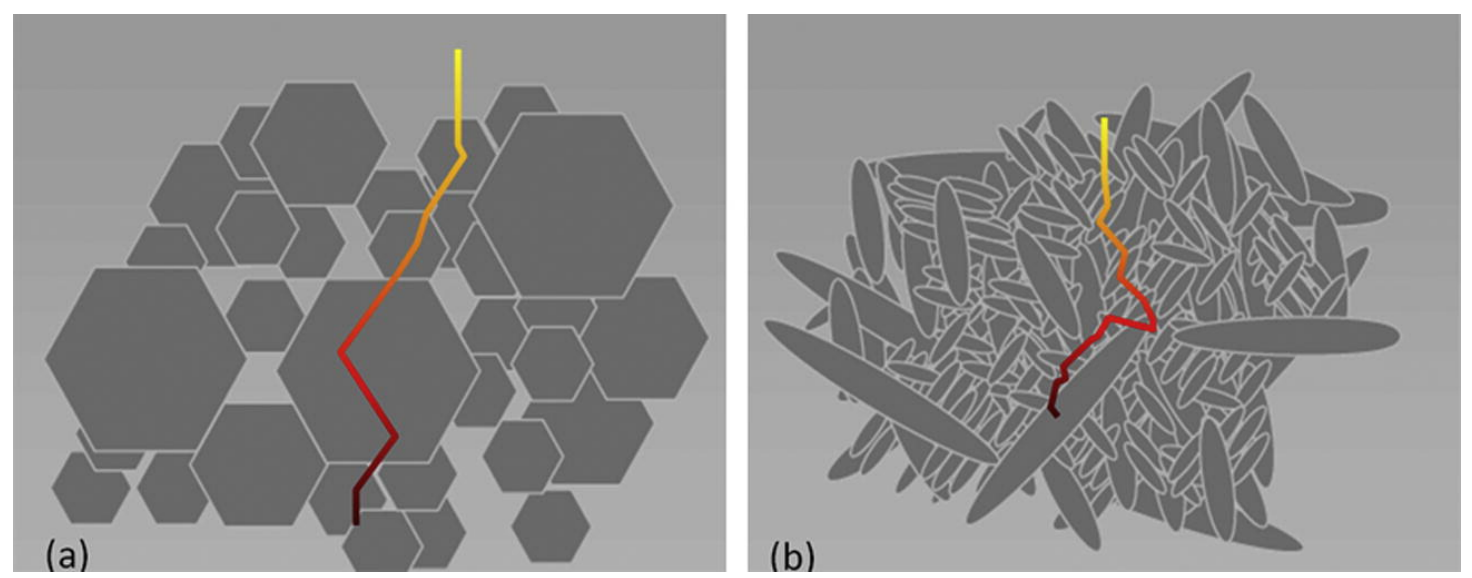

Figure 1.2: An example of the fracture path through a material with a more typical grain structure (a) and through the acicular grain structure of silicon nitride (b). [1]

It has been shown [4] that the behavior of crack propagation in ceramic materials is a key factor in the overall strength of the material; the unique advantages of silicon nitride in this regard make it an attractive material for many novel devices. 


\subsection{Silicon nitride film applications}

The proposed applications for silicon nitride in the wider field of engineering run the gamut, with proposals for its use in combustion engines dating back to 1999 [5]. With the development of micro-electronic mechanical systems, or MEMS, considerable interest has generated around using silicon nitride in these systems as well [6] and [7]. The use of the nitride has even found interest in such unlikely places as in battery technology, where it also appears as a thin coating [8].

In the field of biomedical engineering specifically, silicon nitride finds a great deal of interest. One popular area for proposed uses of silicon nitride is in the construction of orthopedic implants, particularly the articulating surfaces that would normally be subject to a great deal of wear in vivo. Concepts for articulating surfaces that would create low volumetric wear led to the creation and manufacture of metal-on-metal bearing surfaces, but a higher-than-expected occurrence of early failure in these implants was traced back to an accumulation of metallic wear debris [9] and [10]. The cytotoxicity of fine debris from articulating cobalt-chrome surfaces has been demonstrated in vitro [9]. Similar effects have also been tested for titanium wear debris [11]. In both cases, cell lines exposed to the metal debris increased their production of hypoxia-inducible factor 1, a precursor to vascular endothelial growth factor, which has been implicated in aseptic loosening of orthopedic implants [10]. 
With demonstrated detrimental effects from the two most popular orthopedic implant construction materials, researchers interested in minimizing volumetric wear debris have examined silicon nitride for use in this capacity. [12-17]. Neumann et al. demonstrated that silicon nitride is a comparable growth surface to commonly used titanium alloys, in terms of cytotoxicity, cell viability, and cell morphology [12]. Silicon nitride has been reported to possess an antibacterial or bacteriostatic effect, an additional deterrent to implant failure when it is included [16]. When comparing the efficacy of the material as a bearing surface to the metals typically employed in this capacity, silicon nitride has clear

advantages: the wear rate of articulating silicon nitride coatings can be as low as $1.3 \times 10^{-7}$ $\mathrm{mm}^{3} / \mathrm{N} \bullet \mathrm{m}[14]$. Silicon nitride coatings in orthopedic implants may even find use with the traditional ultra-high molecular weight polyethylene bearing surface; besides reducing the volume and the toxicity of the wear debris, the silicon-nitride coating reduces the friction coefficient between the articulating surfaces by as much as one-half when compared to an uncoated cobalt-chrome bearing surface [17]. For these reasons, the use of silicon nitride as a thin coating in implant bearing surfaces is likely to come into effect.

\subsection{Nanoindentation Theory}

Nanoindentation has become the preferred method for testing the mechanical properties of a thin film. The larger-scale method of microindentation was largely surpassed when the depth of the probe could be measured directly, allowing the contact area to be indirectly measured even for very small contact areas. The use of small-scale 
indentations, both in terms of absolute size as well as in magnitude of applied force, allows for precise measurement of very small samples. Nanoindentation produces a loading/unloading curve, where the force applied via the indenter is measured against the depth of the indentation. Because the geometry of the indenter tips used in nanoindentation is known and precise, the depth measurement is sufficient to calculate the total contact area at every stage of the indentation process. The nanoindentation process produces a standard loading-unloading curve that reveals the combination of elastic and plastic processes during an indent. In particular, the initial portion of the unloading curve is recorded while the material is still in its elastic rebound phase. 


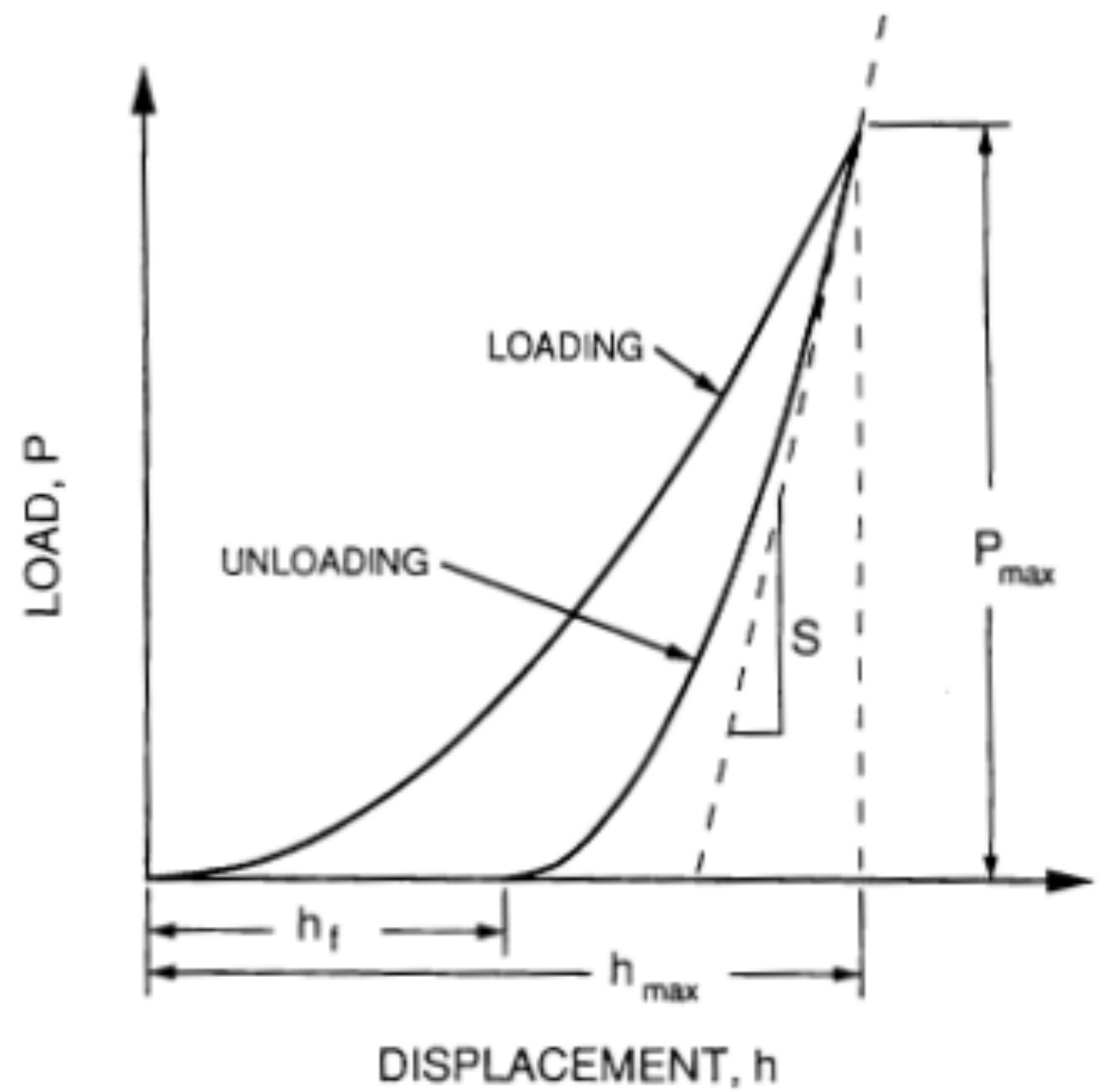

Figure 1.3: An idealized nanoindentation loading-unloading curve. [18]

Interpretations of this curve and the measurements derived from depth-sensing instrumentation have been the subject of much research, but the work by Oliver and Pharr has become the standard in the field [18-21]. Oliver and Pharr's model gives the unloading curve measured during indentation as a power-law function of the following form: [18]

$$
P=l\left(h-h_{f}\right)^{m}
$$


Where $P$ is the load in $\mathrm{mN}, h$ is the instantaneous indentation displacement in $\mathrm{nm}, h_{f}$ is the final indentation displacement in $\mathrm{nm}$, and both $l$ and $m$ are dimensionless fitting parameters.

The slope of this section of the curve is the contact stiffness of the material, $S$. This value can be represented as the rate of change of loading over the rate of change of the depth, which can be related to the contact area and reduced modulus as follows: [18]

$$
S=\frac{d P}{d h}=\frac{2}{\sqrt{\pi}} E_{r} \sqrt{A}
$$

The measured reduced modulus is an indirect measurement of the actual sample modulus being indented. The reduced modulus is a result of the indenter itself not being perfectly rigid: the use of diamond indenters minimizes this effect, but it still needs to be accounted for. The reduced modulus is related to the actual sample modulus via the following expression: [18]

$$
\frac{1}{E_{r}}=\frac{\left(1-\mathbf{v}^{2}\right)}{E}+\frac{\left(1-\mathbf{v}_{i}^{2}\right)}{E_{i}}
$$

Where $v_{\mathrm{i}}$ and $E_{i}$ refer to the Poisson's ratio and the elastic modulus of the indenter. Using this expression, it is possible to determine the modulus encountered with a specific specimen as long as the Poisson's ratio of the specimen is known. 


\subsection{Nanoimpact Theory}

A nanoimpact test is essentially a nanoindentation test taking place at comparatively high speed, repetitively and at a constant frequency, for a given period of time. The same load is applied by each iteration of the test, so the displayed result will be the change in the depth of the material over the time of the nanoimpact test. This result allows the rate of damage, or the rate of change of depth in the material, to be derived from the recorded data. There are two types of nanoimpact test: the pendulum oscillation test, sometimes called multiple impulse; and the sample oscillation test, sometimes simply called impact. The pendulum oscillation test moves the indenter in order to create an impact, while the sample remains stationary. The sample oscillation test moves the sample, while the indenter remains stationary.

\subsection{Nanoscratch Theory}

A nanoscratch test (sometimes referred to as a scanning test) can be thought of as combining the actions of an indentation and a cut through the material in question. The test is carried out using a probe attached to a highly sensitive friction transducer. The stress experienced by the probe tip has two components: the ploughing stress and the shear stress. Ploughing stress is a measure of the transverse stress on the probe tip caused by its motion through the coating, while the shear stress is the stress caused by the contact of the probe tip. 
The work of adhesion of a thin film over a substrate can be found via the following relationship [22]:

$$
W=\frac{1}{2} t \frac{\boldsymbol{\sigma}^{2}}{E_{c}}
$$

Where $t$ is the film thickness and $E_{c}$ is the Young's modulus of the coating.

The Burnett and Rickerby model describes the effects of the ploughing stress and the shear stress on the measured friction force as shown below [23]:

$$
F=A_{1} \boldsymbol{\sigma}_{p}+A_{2} \tau
$$

Where $A_{1}$ is the cross-sectional area of the track and $A_{2}$ is the cross-sectional contact area. Because $A_{2}$ is much larger than $A_{1}$, ploughing stress contributes most to coating failure, and the shear stress term can be ignored when calculating critical load [24]. If the shear stress term is ignored, the ploughing stress can be found from the measured friction force via the relation given below: 


$$
\sigma_{p}=\frac{F}{A_{1}}
$$

Using that expression, the final Burnett and Rickerby model is derived [23]:

$$
L_{c}=\frac{\pi d_{c}^{2}}{8}\left(\frac{2 E W}{t}\right)^{\frac{1}{2}}
$$

where $d_{c}$ is the track width and the diameter of the contact area, $t$ is the coating thickness, and $\mathrm{E}$ is the coating's Young's Modulus.

In a 1988 paper, Bull et al suggested a refinement of that model with added terms for the friction coefficient and Poisson's ratio of the coating that appears as follows [25]:

$$
L_{c}=\frac{A_{1}}{\mathbf{v} \boldsymbol{\mu}_{c}}\left(\frac{2 E W}{t}\right)^{\frac{1}{2}}
$$

Here, $A_{1}$ is found by the following expression [25]:

$$
A_{1}=R^{2} \sin ^{-1}\left(\frac{d}{2 R}\right)\left\{\frac{-d}{2}\left[R^{2}-\left(\frac{d}{2}\right)^{2}\right]^{\frac{1}{2}}\right.
$$


Where $\mathrm{R}$ is the indenter radius and $\mathrm{d}$ is the width of the scratch track.

In their 1996 paper, Attar and Johannesson proposed a different modification to the Burnett and Rickerby model, where the stress responsible for the coating failure would be expressed as [24]:

$$
\sigma=\frac{\mathbf{v} \boldsymbol{\mu}_{c} L_{N}}{d t}
$$

Where $\mathrm{L}_{\mathrm{N}}$ is the normal load on the coating, $\mathrm{t}$ is the coating thickness, and $\mathrm{d}$ is the scratch track width. By using this expression for stress in the Burnett and Rickerby model, the modified critical load model proposed by Attar and Johannesson becomes [24]:

$$
L_{c}=\frac{d_{c}}{\mathbf{v} \boldsymbol{\mu}_{c} L_{N}}(2 t E W)^{\frac{1}{2}}
$$

So, by combining measured friction forces obtained via the friction transducer with depth measurements, the known normal load at which the coating began to fail, the width of the scratch track at that point, and material properties of the coating (Poisson's Ratio, Young's Modulus) the stress and critical load values for the coating can be found. 


\subsection{Research Objectives}

The purpose of this research project was to investigate the use of nano-scale material testing techniques to quantitatively evaluate the material properties, quality, and consistency of thin ceramic films. The specific test types used in this study were nanoindentation, nanoimpact via sample oscillation and pendulum oscillation, and nanoscratching. As academic and industrial interest in the use of ceramic thin films increases, techniques for the validation of such films on a small scale will prove valuable.

The ability to measure the elastic modulus and hardness of the ceramic coating through nanoindentation allowed evaluation of the film properties at various points on the entire sample. The two nanoimpact techniques, which compare the depth of the damage to the number of applications of a known force value through either method, likewise allow the film integrity to be evaluated as well as allowing for a comparison of the film's reaction to the two forms of impact damage. Finally, the use of the nanoscratch test allows for the evaluation of the film's work of adhesion to its substrate. From this an the other data gathered during the scratch tests, the critical load of the film across wafers and different testing parameters can be compared.

The following hypotheses are addressed in this study:

Hypothesis 1: The silicon nitride thin films will have highly consistent hardness and elastic modulus as measured by nanoindentation, both between wafer quadrants and 
between wafers. The nature of the fabrication process for the nitride thin films should prevent significant deviation.

Hypothesis 2: The silicon nitride thin films will be more resistant to fracture from pendulum oscillation than from sample oscillation, as measured by the damage rate experienced by the coating due to each test type. Although the initial force experienced by the sample is identical in both cases, the higher frequency of the sample oscillation technique will result in damage to the film structure accumulating at a higher rate.

Hypothesis 3: The critical load of the silicon nitride thin film on its' substrate will be consistent between all test parameters. Changing the testing parameters should not create inconsistencies with the film properties. 


\subsection{Materials and Methods}

\subsection{Materials}

The silicon nitride used for testing needed to be in the form of a thin film of the same size as would be deposited or grown onto a device's surface. Three silicon wafers with a 2000

$\AA$ thick coating of silicon nitride were obtained for use as the testing material. The wafers had a standard diameter of $100 \mathrm{~mm}$. The wafers were p-type doped silicon, with a 110 crystallographic orientation. Each wafer had a nominal thickness of $500 \mu \mathrm{m}$, including the 2000 A silicon nitride coating.

The large diameter of the wafers made them unsuitable for testing as a complete unit. In order to prepare the samples that were used in the tests, each wafer was first scored and broken into quadrants, using the 110 crystallography to assist with breaking the wafers into straight-edged pieces. 


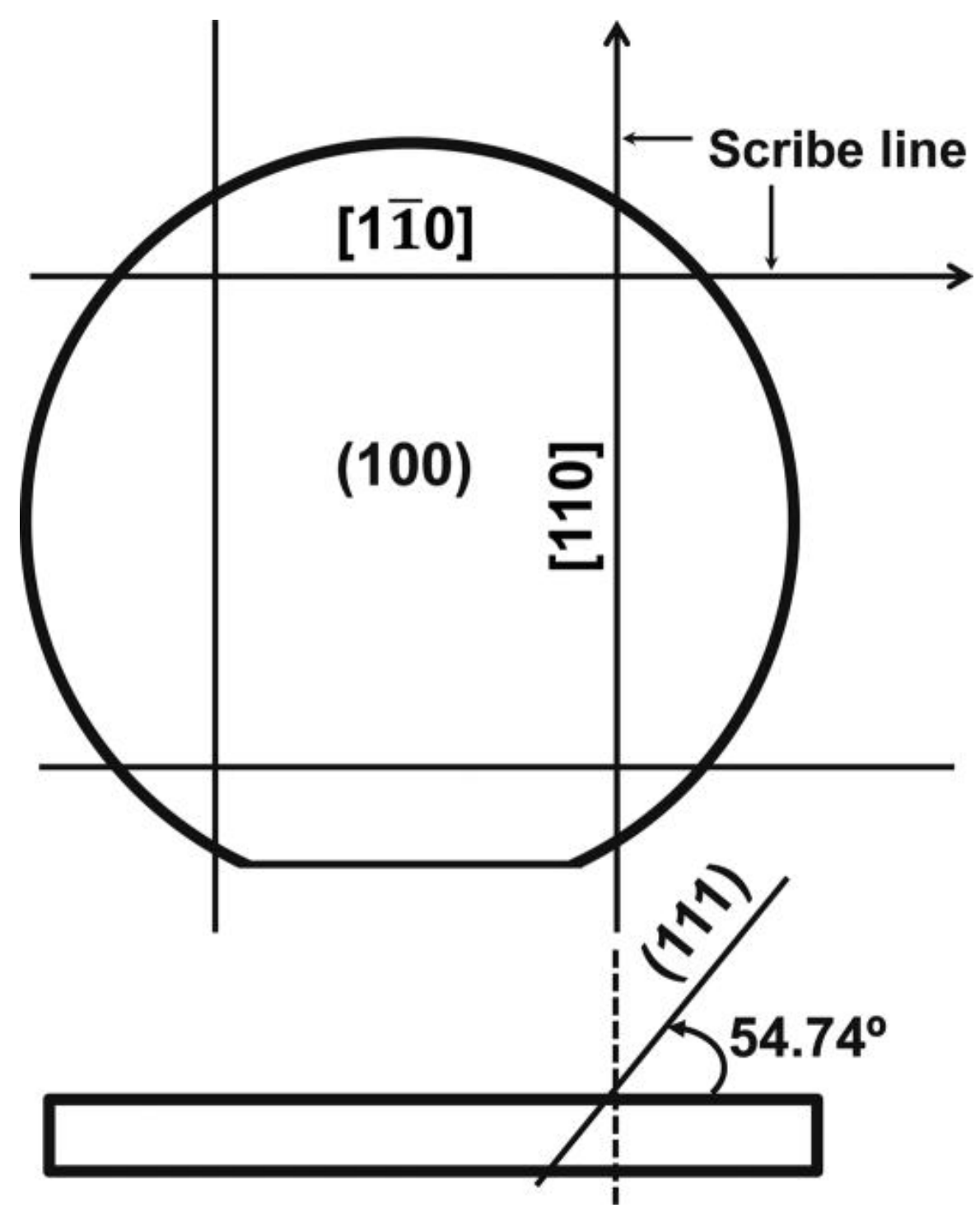

Figure 3.1: The crystallographic planes of a $<110>$ orientation silicon wafer. [26]

One quadrant of wafer 3 was used to create test samples, which were used both for machine calibration and to determine whether a $2 \mathrm{~cm} \mathrm{X} 2 \mathrm{~cm}$ or a $1 \mathrm{~cm} \mathrm{X} 1 \mathrm{~cm}$ sample was preferable. The ease of use in the machine and the number of samples that could be prepared from each wafer quadrant were the primary factors evaluated to make the decision regarding sample size. The $1 \mathrm{~cm}$ X $1 \mathrm{~cm}$ sample size was chosen because more 
samples could be taken from each quadrant of the wafers, and because the sample would be fully supported by the sample mount. With the sample size decided, the quadrants of the wafers were used to prepare 8 specimens of approximately $1 \mathrm{~cm}^{2}$ size. Samples could not be prepared to uniform dimensions due to the scoring and breaking method employed. The deviation from the ideal sample size was considered inconsequential due to the very small area required to perform testing. Once the samples from a quadrant had been prepared, one corner of the sample was removed to distinguish that corner. The samples from each quadrant were then stored together in clean petri dishes.

The prepared samples were then labeled on the side without the silicon nitride coating. Labeling was carried out so that the samples could be identified when mounted for testing. Labels consisted of three number designating the wafer of origin, quadrant of origin, and individual sample number. For example, the fourth sample from the second quadrant of the first wafer would be labeled "124". All 72 samples were labeled using this convention. Additional reserve samples prepared from the same wafer stock were also labeled using the convention.

Nanoindentation testing required the samples to be held stationary during the testing process. The nanoindenter sample stage can be locked into position, with a protruding threaded shaft designed to accept a sample stub with a threaded socket in the base. These sample stubs are aluminum cylinders with a height of $6 \mathrm{~cm}$ and a diameter of $2.5 \mathrm{~cm}$. The stubs feature a frontal area that can be used for mounting samples up to $4.9 \mathrm{~cm}^{2}$, which was sufficient to completely support the approximately $1 \mathrm{~cm}^{2}$ samples described above. 
Prior to each test, a sample was selected and the identity of the sample was recorded. A small amount of cyanoacrylate glue was used to securely anchor the sample to the sample stub. Using nitrile gloves, the samples were firmly pressed flat against the top surface of the mounting stubs. The samples were oriented on the stubs with the blunted corner created during sample preparation on the bottom left of the mounted sample. After orientation, the glue was allowed to completely cure before the sample stub was affixed to the sample stage.

\subsection{Device and Device Calibrations}

Testing was carried out using a NanoTest NTX (Micro Materials Ltd., Wrexham, UK). This system is capable of carrying out tests in a variety of ways, including indentation, impact with either sample oscillation or pendulum oscillation, and scanning in both openair and fluid-immersed environments. These tests can be performed using a low-load testing sensor, referred to hereafter as the nanotest module, or a high load-testing sensor, referred to hereafter as the microtest module. While both the microtest and nanotest modules were available, only the nanotest module was used to collect data. The NTX used for testing is kept in a temperature-controlled cabinet, with the cabinet air temperature maintained at $22^{\circ}$ Celsius during test runs. Additionally, the room containing

the indenter cabinet featured a long-duration temperature control system, which was used during testing to maintain an ambient temperature of $22^{\circ}$ Celsius. The indenter cabinet contained a vibration-isolating table, which the indenter was completely supported by. The vibration isolation table was deemed necessary due to the sensitivity of the 
equipment and the low force levels that the testing was carried out at. The indenter used in the study also featured a remotely operated turreted microscope with magnification options ranging from 40 to $400 \mathrm{x}$. The microscope was primarily used for the alignment of the sample relative to the indenter, as well as for the positioning of the indenter prior to the beginning of the experiment sequence.

The indenter tip used with the nanoindentation module of the NanoTest NTX described above was changed depending on the type of test that was going to be carried out. All indenter tips contained a small diamond tip with a specific geometry corresponding to the selected test. The interchangeable indenter tips took the form of a steel mounting block with the same geometry as the smaller diamond contact tip. The larger steel mounting blocks have a long, thin steel rod on the face opposite of the diamond contact tip. This steel rod would be inserted into the pendulum of the nanotest module, and then held in place with a small brass screw. 


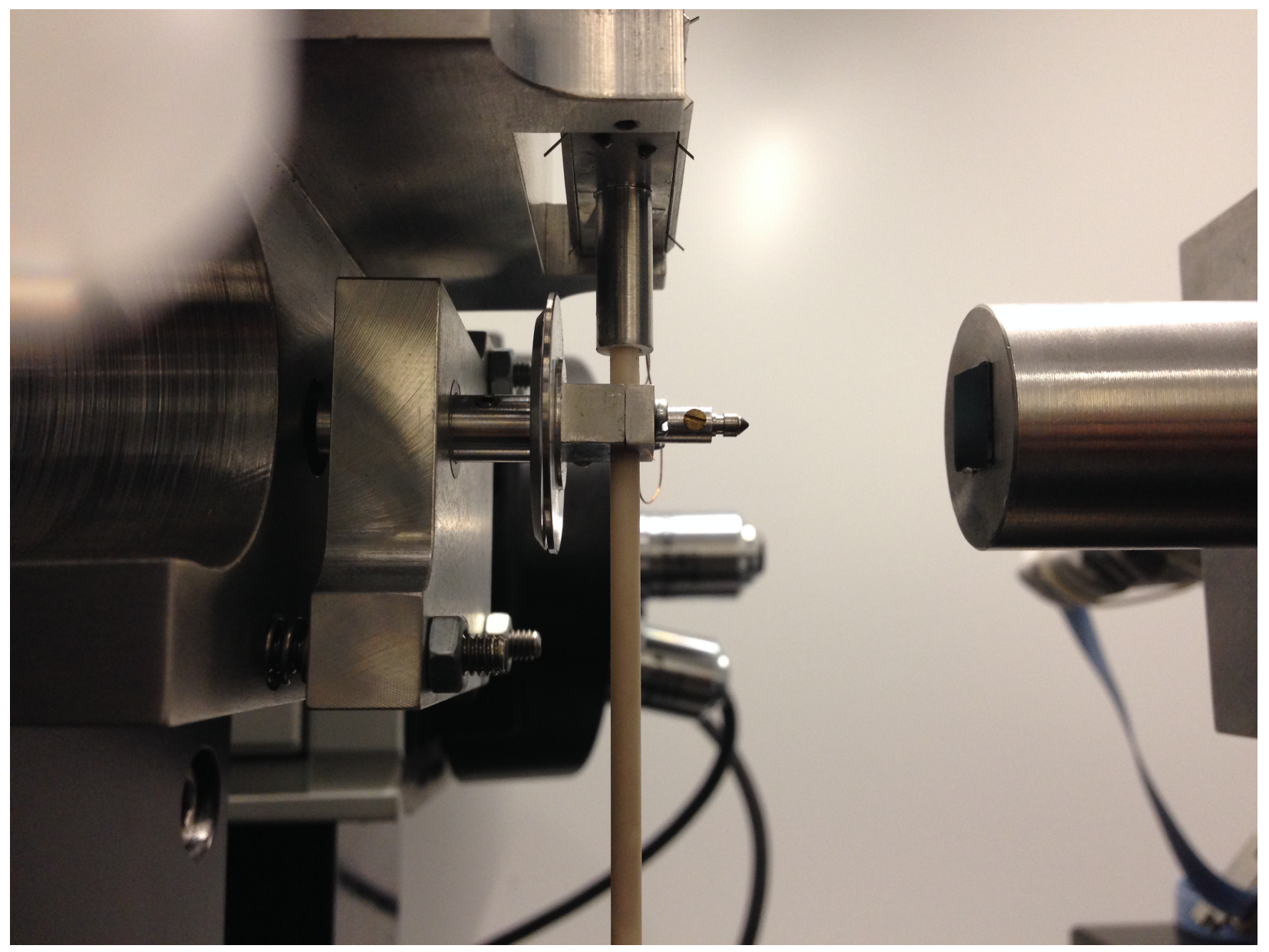

Figure 3.2: A Berkovich indenter tip in the NanoTest module. The pendulum is to the left of the indenter tip.

For nanoindentation tests, the indenter tip used was a Berkovich diamond tip, consisting of a three-sided pyramidal section with an axis-to-face angle of 65.27 degrees. For the nanoimpact tests, both sample oscillation and pendulum oscillation, a cube corner tip was used. This tip is a section of a cube with an axis-to-face angle of 35.26 degrees. For nanoscratch tests, the indenter used had a conical geometry. The selection of the conical geometry was based on the consideration of uneven wear to the corners and faces of the other indenter tip options if used to create a lateral scratch in a material. A conical tip is 
not completely immune to this effect, but the lack of sharp and defined features means that the degradation resulting from the test is considerably more even and less likely to produce a significant effect on the volume or integrity of the indenter tip.

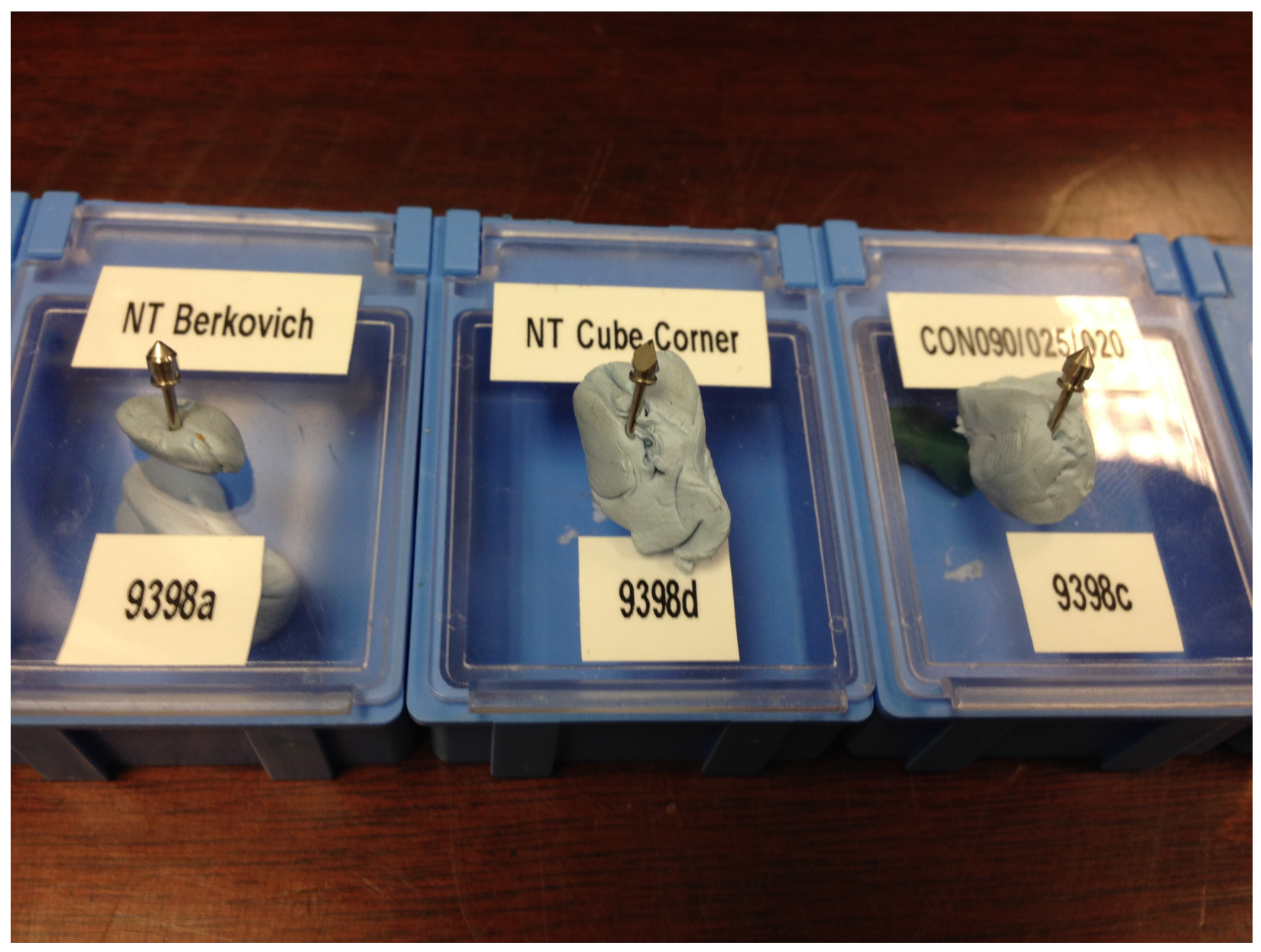

Figure 3.3: The selection of indenter tips used in this study. From left to right: Berkovich, Cube Corner, and Conical.

Prior to carrying out any testing using the indenter, multiple machine calibrations were performed to ensure the accuracy of the data being gathered. The frequency with which calibrations were carried out varied over the course of data collection. Some calibration 
procedures were necessary with high frequency, up to once per day. The most pertinent calibrations are described here in detail.

The nanoindenter used for these tests featured a bridge box that was used to determine how much flexion the pendulum was experiencing. As the pendulum moves, the separation of a pair of plates in a parallel plate capacitor changes, resulting in a change in the output voltage from a capacitance bridge. This allows the displacement of the diamond indenter tip to be measured, because one of the plates in the capacitance bridge is connected to and allowed to move with the mounting device for the diamond indenter. Calibration of the bridge box was carried prior to data collection to ensure that measurements of the indenter tip displacement would be as accurate as possible.

The pendulum determines loads based on a known relationship between the load applied to the pendulum and the change in the output voltage. The load calibration uses known test masses to verify that the data is correct. Masses with values of $1.090 \mathrm{~g}, 2.032 \mathrm{~g}$, and $2.975 \mathrm{~g}$ were sequentially suspended from a mounting point on the pendulum, and 16 load calibrations would be performed with the known mass to establish the response curve for that loading. After the calibrations for one mass were complete, the mass was replaced with the next largest one until 48 calibrations had been made. At this point, the load calibration is complete. Load calibrations were conducted once every one to two weeks that data collection was taking place. 
The depth calibration is used to relate the position of the sample stage motors to the depth of the probe in contact with the sample being tested with the nanoindentation module. To perform this calibration, a reference sample of fused silica is brought into contact with the probe. The capacitance between the two plates of the pendulum is then compared to the position of the sample stage as reported by the motor encoders. The calibration allows the amplifier gain of the system to be adjusted as well, effectively increasing the range of possible sensitivities and therefore measurable depths that the system can accommodate. Since the depth of the indenter needs to be known to calculate the contact area, this is a crucial calibration when using the nanoindentation module.

The positioning of the sample stage was important throughout the data collection process. The location of the focal plane was a useful piece of information so that a minimum of focusing work was required after a testing series. The yz lateral position of the sample stage must be kept consistent to allow for visual inspection of the test sites with the optical microscope after a test series. The sample stage calibration involved measuring the distance from contact with the indenter tip to the focal plane. Each test series would end with the indenter tip just barely out of contact with the surface, so with the necessary retraction distance known to the NanoTest platform, the stage could be automatically retracted to the correct distance for optical microscope survey after each test series. The yz position calibration used a reference point on the surface of the sample coupled with a crosshairs superimposed over the optical microscope image. The crosshairs and the reference point were aligned to keep the yz-displacement as low as possible. The sample 
stage calibration was also conducted infrequently, with about 1 or 2 calibrations per week of data collection.

The frame compliance calibration was completed exactly once during data collection. This was required after a full servicing of the NanoTest system, to establish whether the value had been changed because of modifications to the machine, such as changing or replacing components. The frame compliance value essentially exists as a correction factor to account for the particular characteristics of the individual NanoTest system.

The diamond area function relates to the area of the indenter in contact with the sample during testing. This value would be changed each time the indenter was changed, but the calibration was only performed one time. When the older Berkovich diamond tip used for the nanoindentation testing was removed and replaced with a newer, sharper Berkovich tip, the diamond area function for the Berkovich tip was found and that newer DAF value was subsequently used when indentation testing was carried out.

\subsection{Test types}

The first test series carried out for this study was nanoindentation. Nanoindentation testing was performed with a Berkovich diamond tip indenter in the nanotest module. Using the optical turret microscope at $400 \mathrm{X}$ power, the bottom-left corner of the sample being used for the test would be found. Using this location as the origin, the sample stage would be moved until a suitable starting location was found. The necessary length of the 
planned indentation series would be noted, and this distance and direction away from the designated start point would be checked with the optical microscope. Once it was determined that the indentation path was clear of surface defects that could potentially have different material properties than the rest of the silicon nitride film, the indentation sequence could begin. The indenter tip would remain stationary as the sample surface was brought into contact with it, and the specified load applied. The load on the indenter tip as well as its displacement was measured and recorded during this process in order to generate the depth and load curve for the test. A typical nanoindentation test result appears below: 


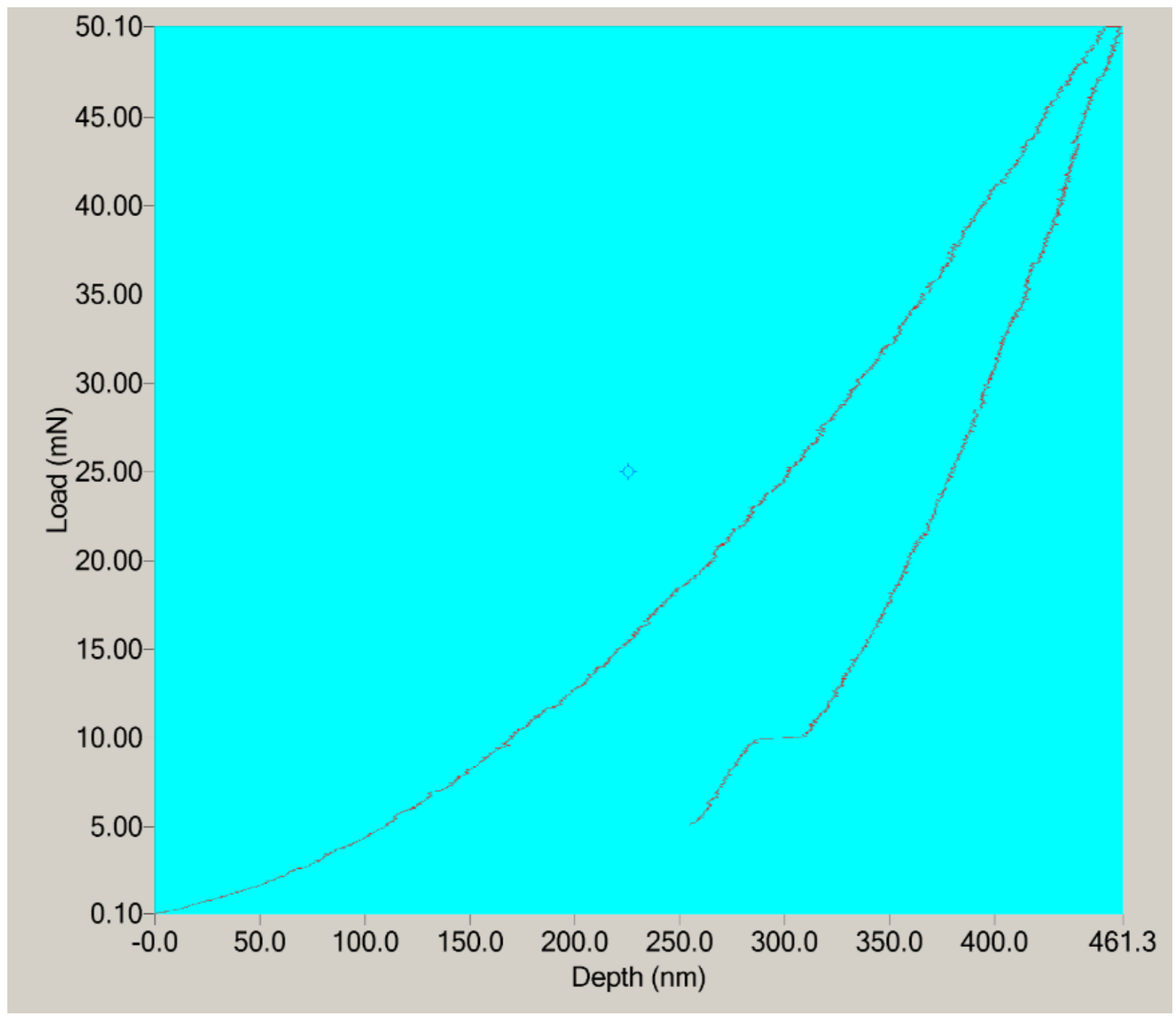

Figure 3.4: A typical nanoindentation test result, displaying a depth and load curve.

Occasionally, environmental factors would cause a data point to be recorded incorrectly. These points were excluded, and replacement tests were conducted to gather valid data. An example of a failed nanoindentation point is shown below: 


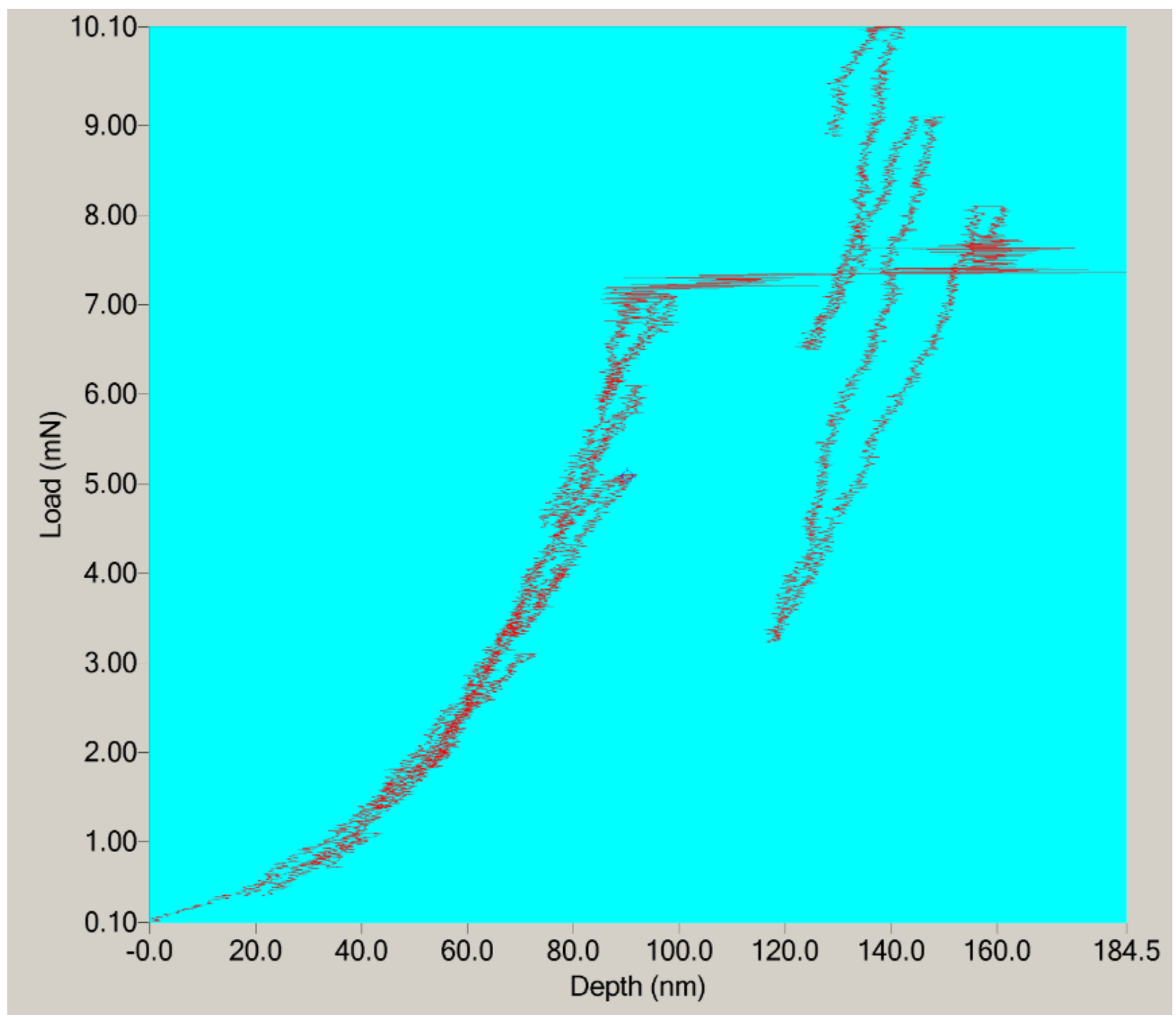

Figure 3.5: A failed nanoindentation. Note the random and meaningless depth measurements.

The second test series carried out for this study was pendulum oscillation impact testing. Pendulum oscillation impact testing uses a cube corner indenter tip. Unlike the nanoindentation testing, the indenter tip does not remain stationary in this test. Instead, a solenoid mounted to the vibration isolation table was brought forward on a micrometer screw. When power was supplied to the solenoid, the solenoid would interact with a ferrous piece on the bottom of the pendulum. When the power to the solenoid was on, the 
pendulum would be drawn back a preset distance. When the power to the solenoid was shut off, the pendulum would swing forward, causing the diamond indenter tip to impact the sample surface. In this way, he behavior of the sample under an external impact could be tested. An example of a nanoimpact data point is given below:

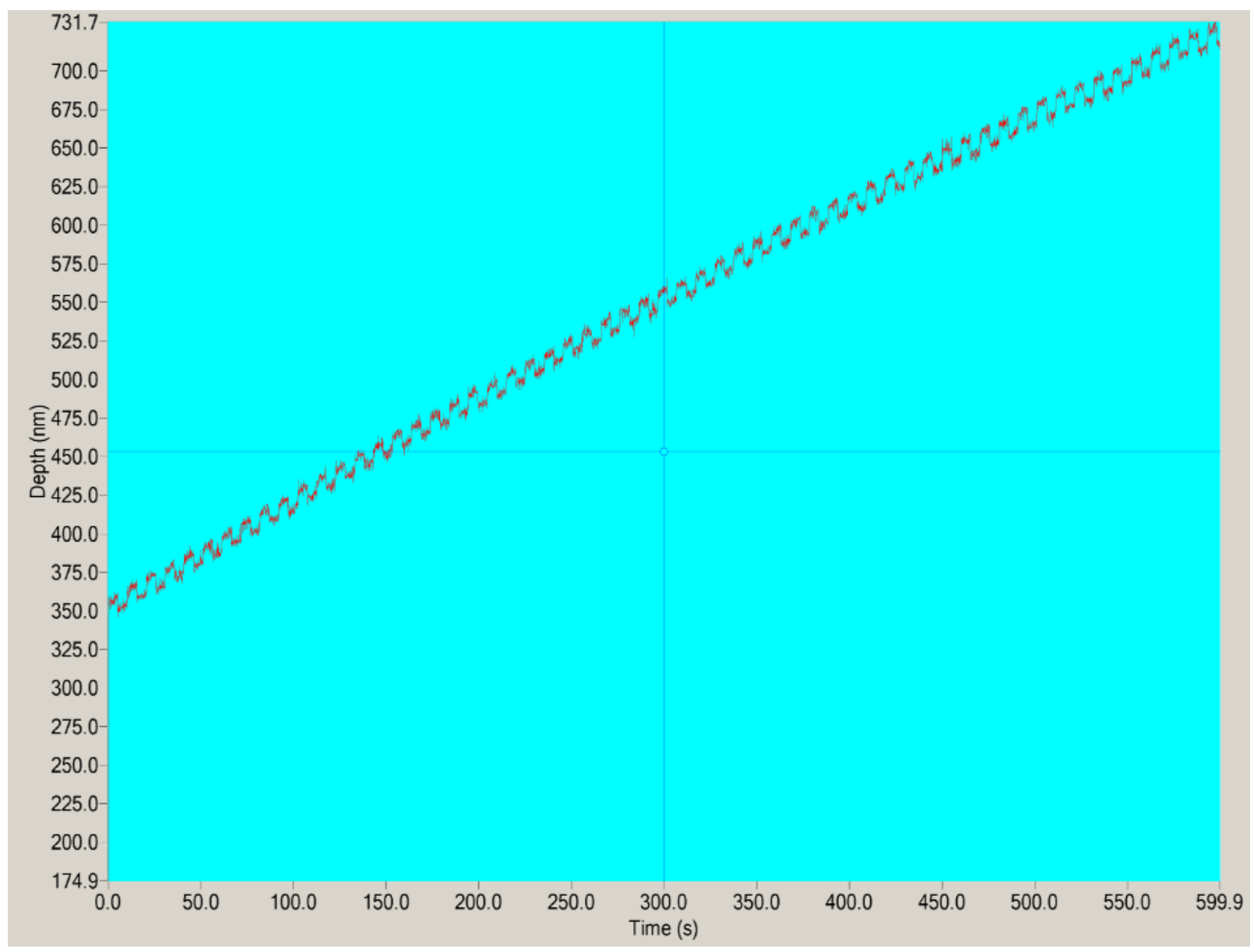

Figure 3.6: A typical nanoimpact result, with the measured depth increasing on each impulse. 
Environmental factors could also cause nanoimpact data points to return bad results.

These points were excluded from data analysis. An example of a failed nanoimpact point is given below:

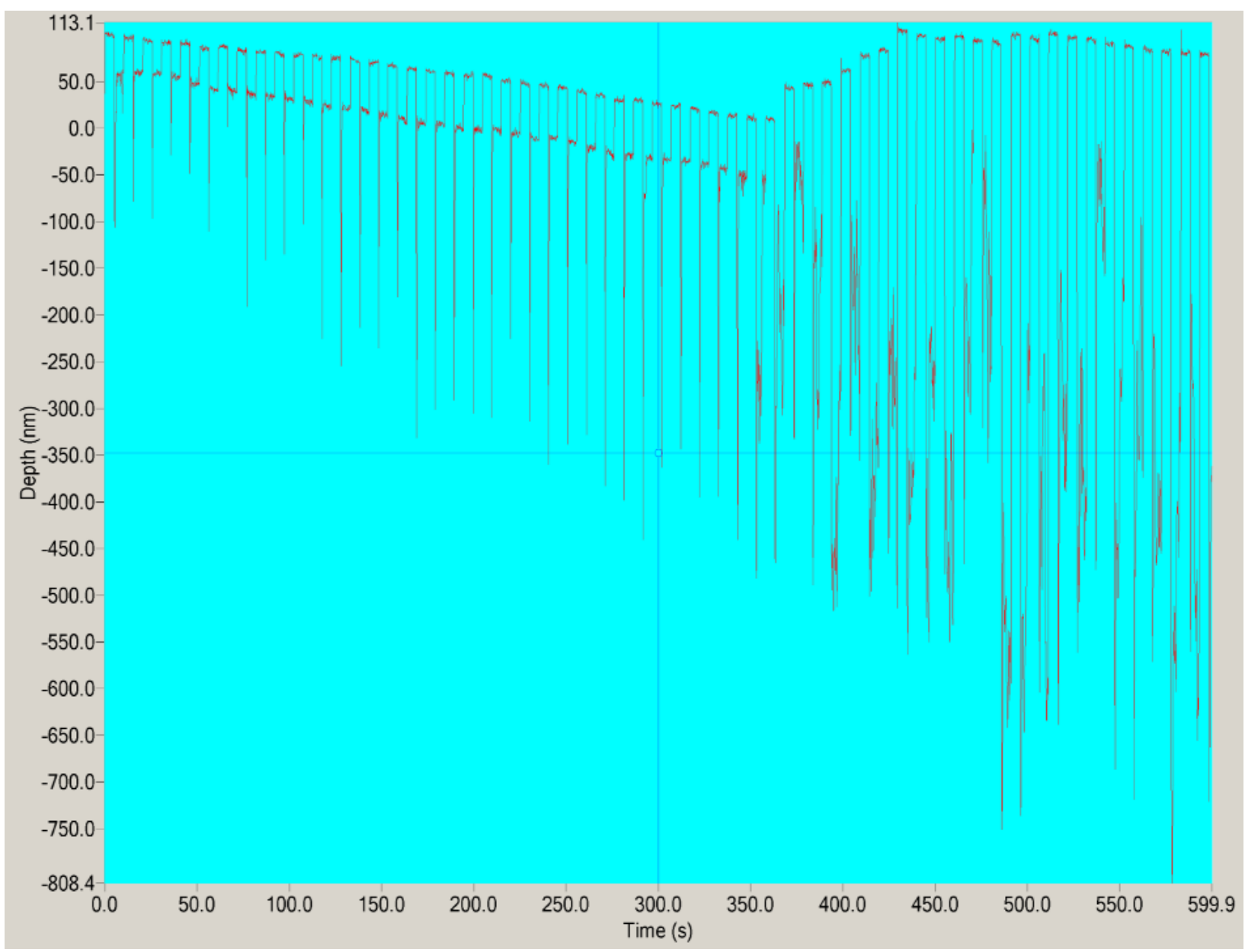

Figure 3.7: A failed nanoimpact result.

The third test series carried out for this study was sample oscillation impact testing. Sample oscillation impact testing uses the same cube corner indenter tip as used in pendulum oscillation impact testing. Unlike in the pendulum oscillation impact testing, the pendulum and the attached indenter tip are stationary. Instead of motion coming from 
the pendulum, the sample is mounted on a piezoelectric stack that is attached to the sample stage in the same way that a normal, inert sample mount would be. The piezoelectric stack oscillates when a periodic signal (square wave) is applied to it. Modifying the voltage and frequency of the signal allows the rate of oscillation to be controlled. By bringing the sample mounted on the stack close to the stationary indenter, the sample can be repeatedly impacted into the indenter tip, allowing data on the behavior of the sample under these conditions to be tested.

The final testing type carried out on the silicon nitride samples was nanoscratch testing. The previous three testing types involved different methods of producing damage on the surface of the material using forces applied perpendicularly to the silicon nitride coating. The nanoscratch test, conversely, begins with a perpendicular force application to push the indenter tip through the coating surface, followed by dragging the indenter across the sample surface. A device called a friction bridge is installed between the pendulum and the conical indenter tip. This device uses changes in resistance through two thin wires during the testing phase to measure the degree of deflection, and therefore implicitly the friction, experienced by the indenter tip during the test. The test type used in this study was the Multipass Wear Test, which consisted of a non-damaging topographical scan, followed by a scratch, and concluding with another non-damaging topographical scan. An example of a nanoscratch test result appears below: 


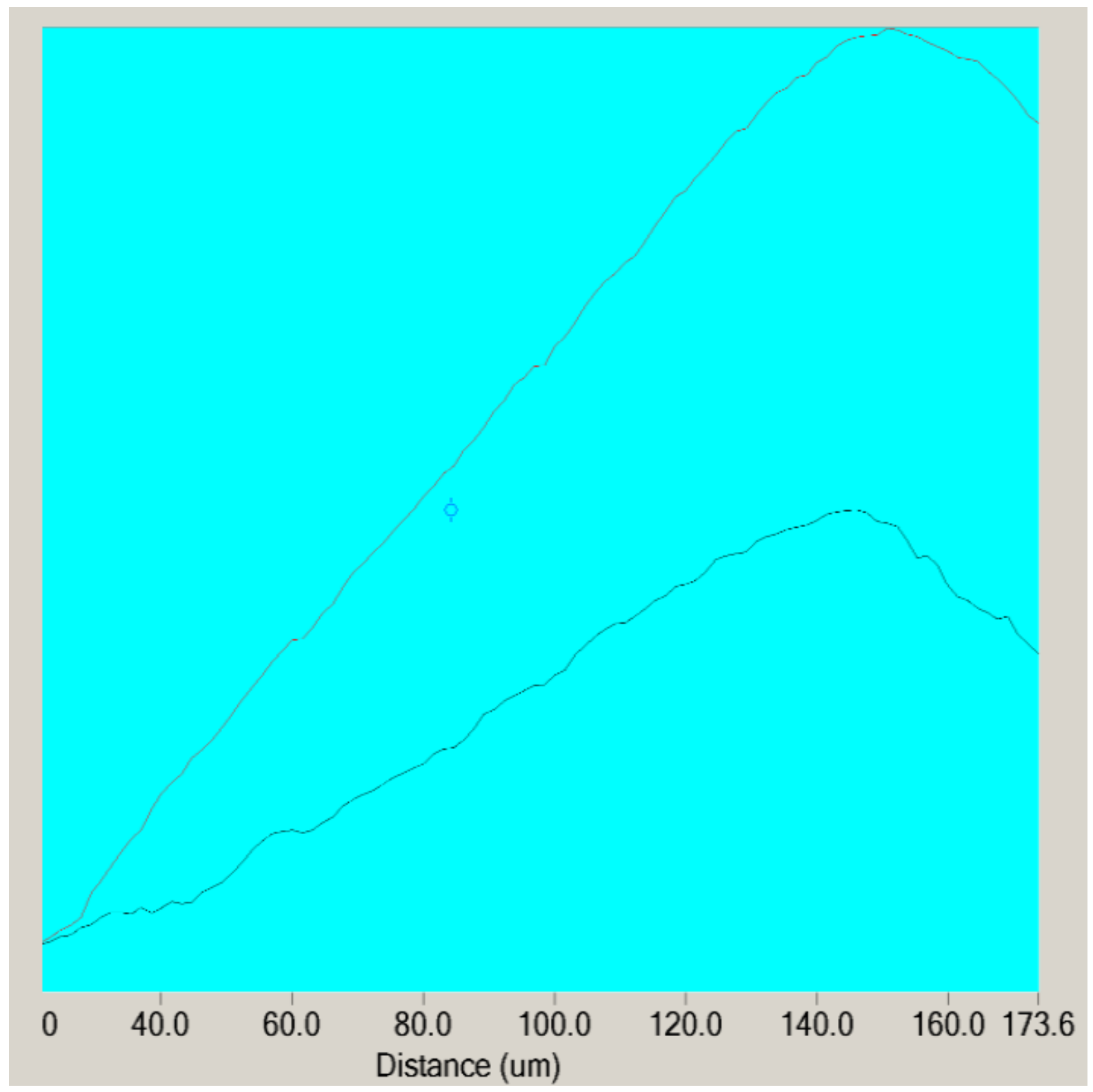

Figure 3.8: A typical nanoscratch result. The upper line is the load, while the lower line is the topographical trace. 


\subsection{Results}

Over the course of the study, 4 different types of test were performed on samples from 3 separate wafers with a silicon nitride coating. In this section, the results for each test type will be analyzed and presented by test type.

\subsection{Microscope Evaluation}

The Nanotest NTX system is equipped with an optical microscope turret that is critical to the operation of the device. Prior to any test being carried out, the microscope was used to check the surface of the test sample for any obvious flaws or atypical areas that could influence the test results. The presence of any abnormalities would result in the test start point being moved to a location where the test could be carried out without the possibility of interaction with any visually apparent flaws. The microscope turret also allowed for visual evaluation of test sites to a limited degree. Indents and impact sites were typically visible and could be imaged with good results. Unfortunately, even under very high magnification levels the results of nanoscratch tests were difficult to image. Although the presence of the scratch could be seen when operating the nanotest, saving the image of the microscope feed made the scratch nearly impossible to see.

In an effort to obtain a clearer image of the nanoscratch results, a sample that had a scratch performed on it was prepped for scanning electron microscopy. The sample was sputtered with $50 \mathrm{~nm}$ of aluminum in argon plasma. Although the precise location of the 
scratch test from a marked corner of the sample was known, the scanning electron microscope was still unable to produce a clear image of the nanoscratch.

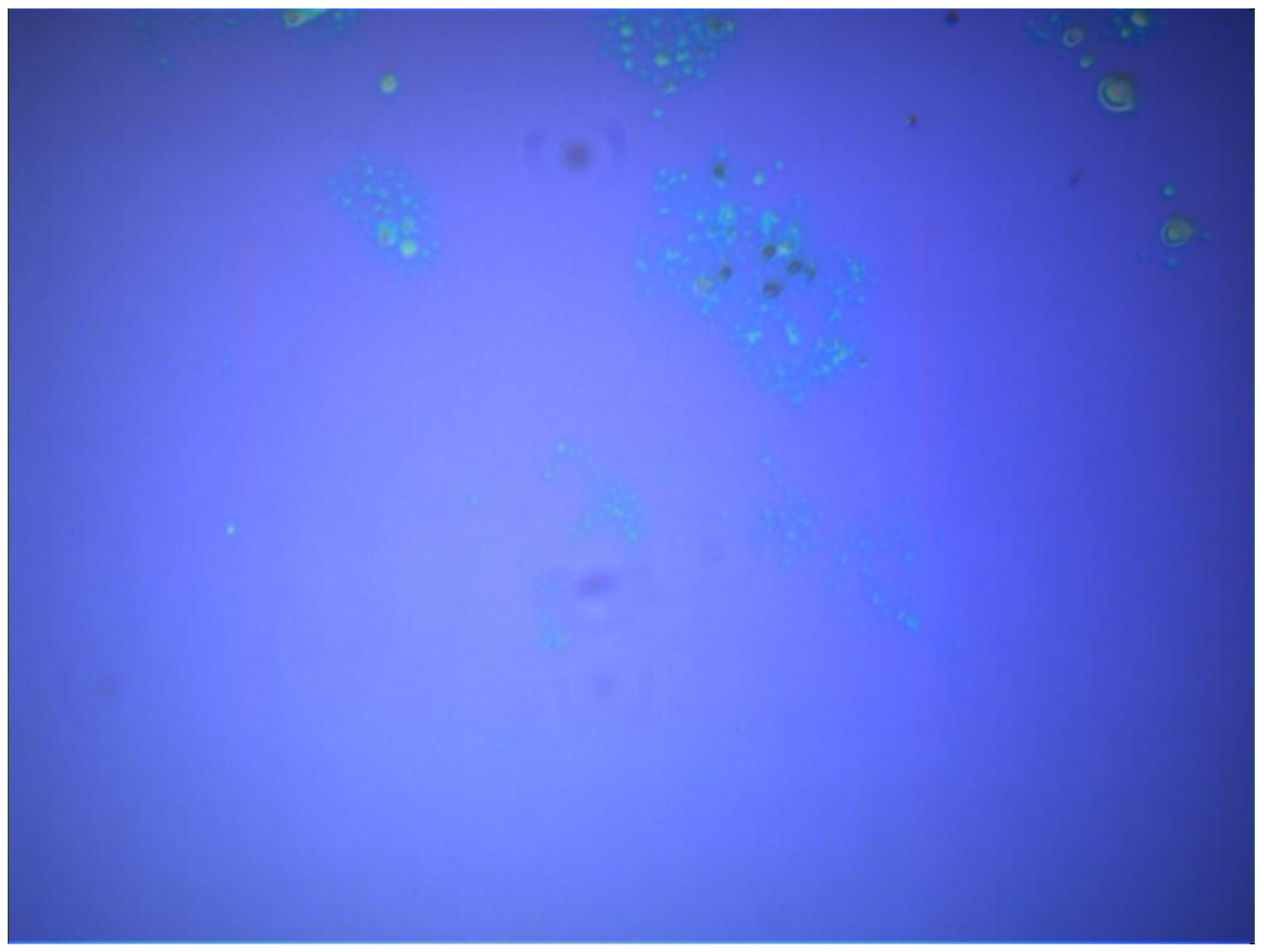

Figure 4.1: An example of the appearance of surface flaws under high magnification. These were avoided when setting up tests in order to control extraneous variation. 


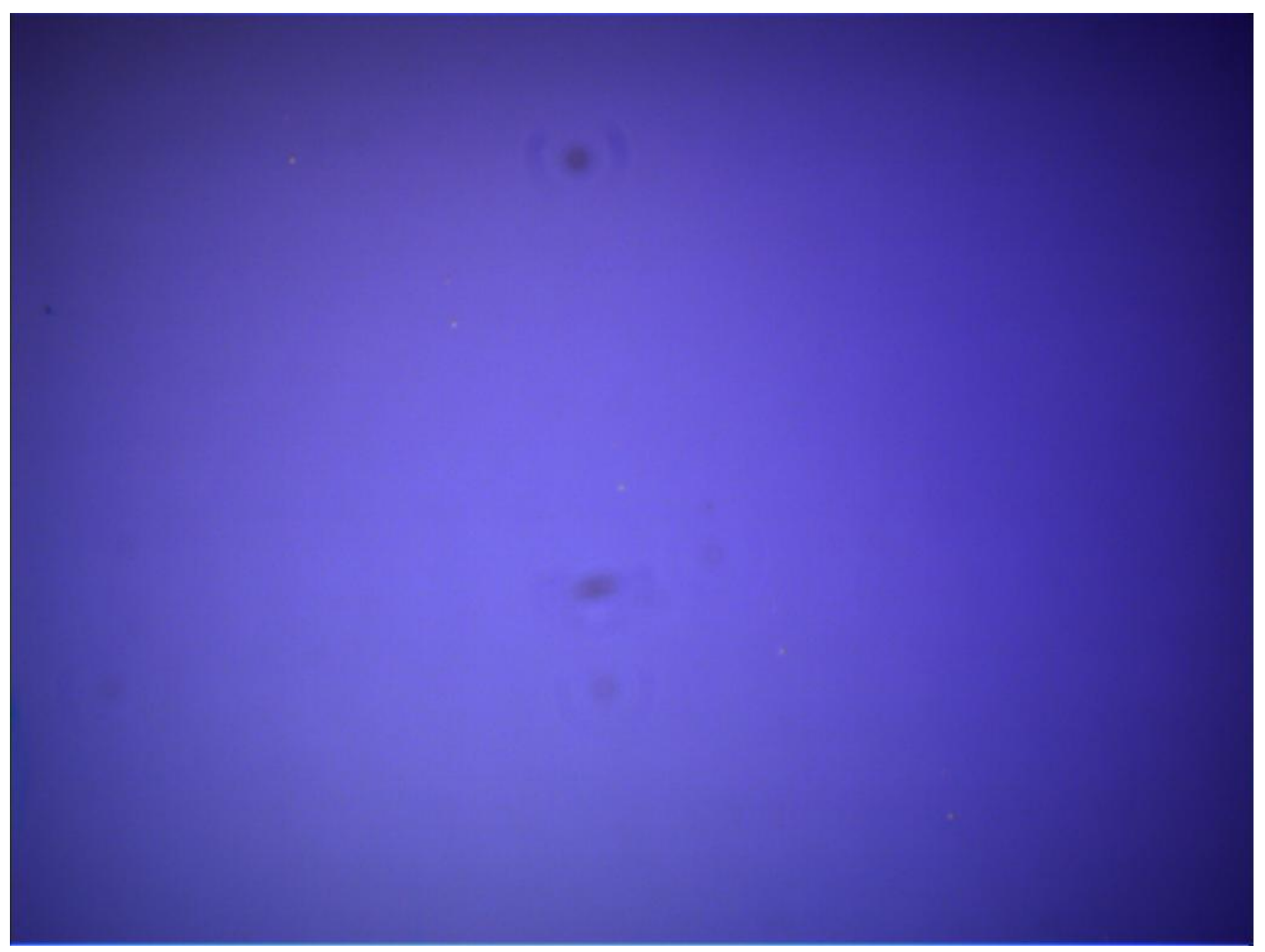

Figure 4.2: An example of a nanoindentation test. The row of indents runs diagonally from the upper left to the lower right and appears as green triangles against the purple nitride background. 


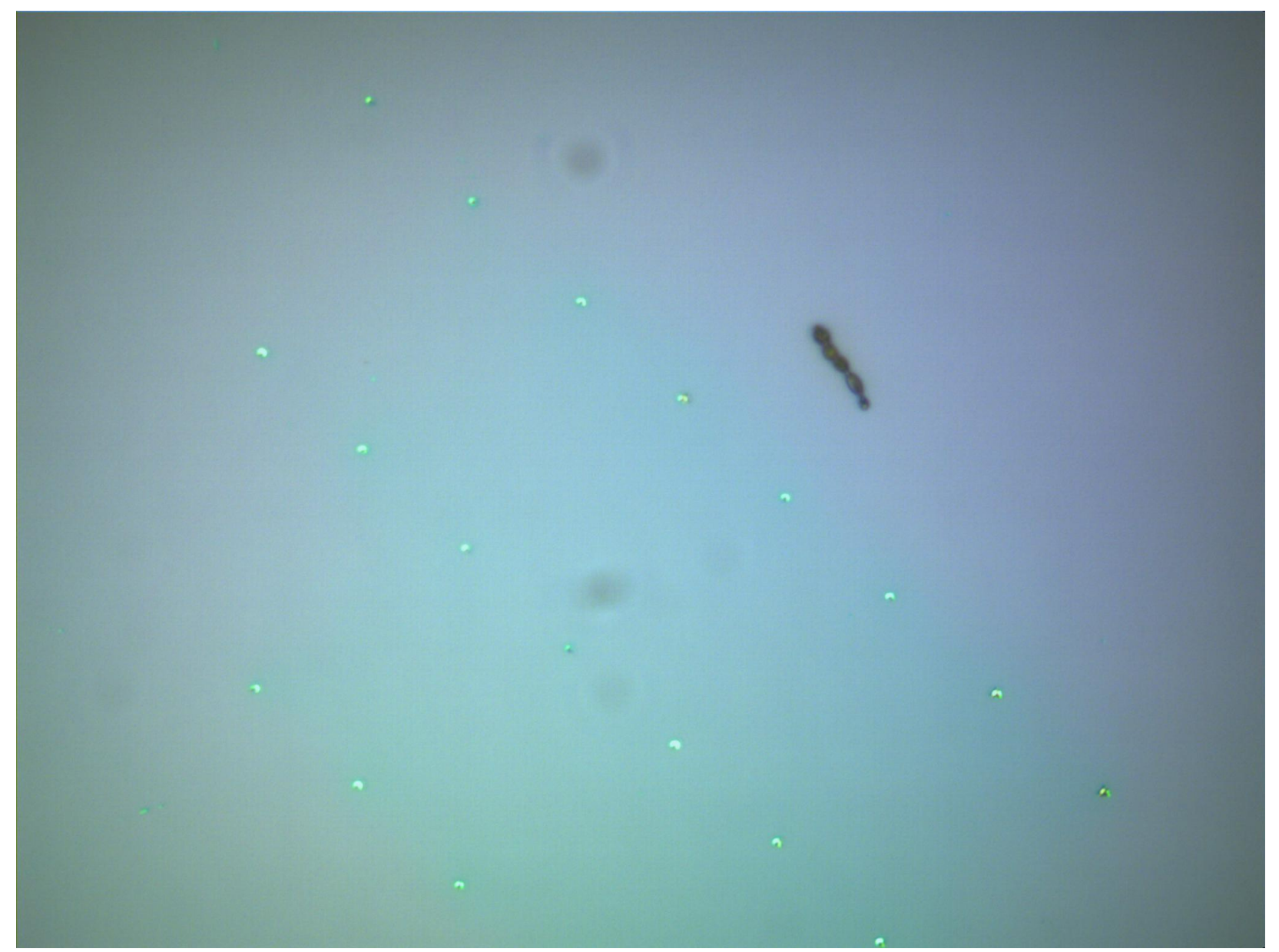

Figure 4.3: Impulse tests carried out for calibration in the same format as the data tests. The impulse sites appear as irregular green dots in diagonal rows from the upper left to the lower right. 


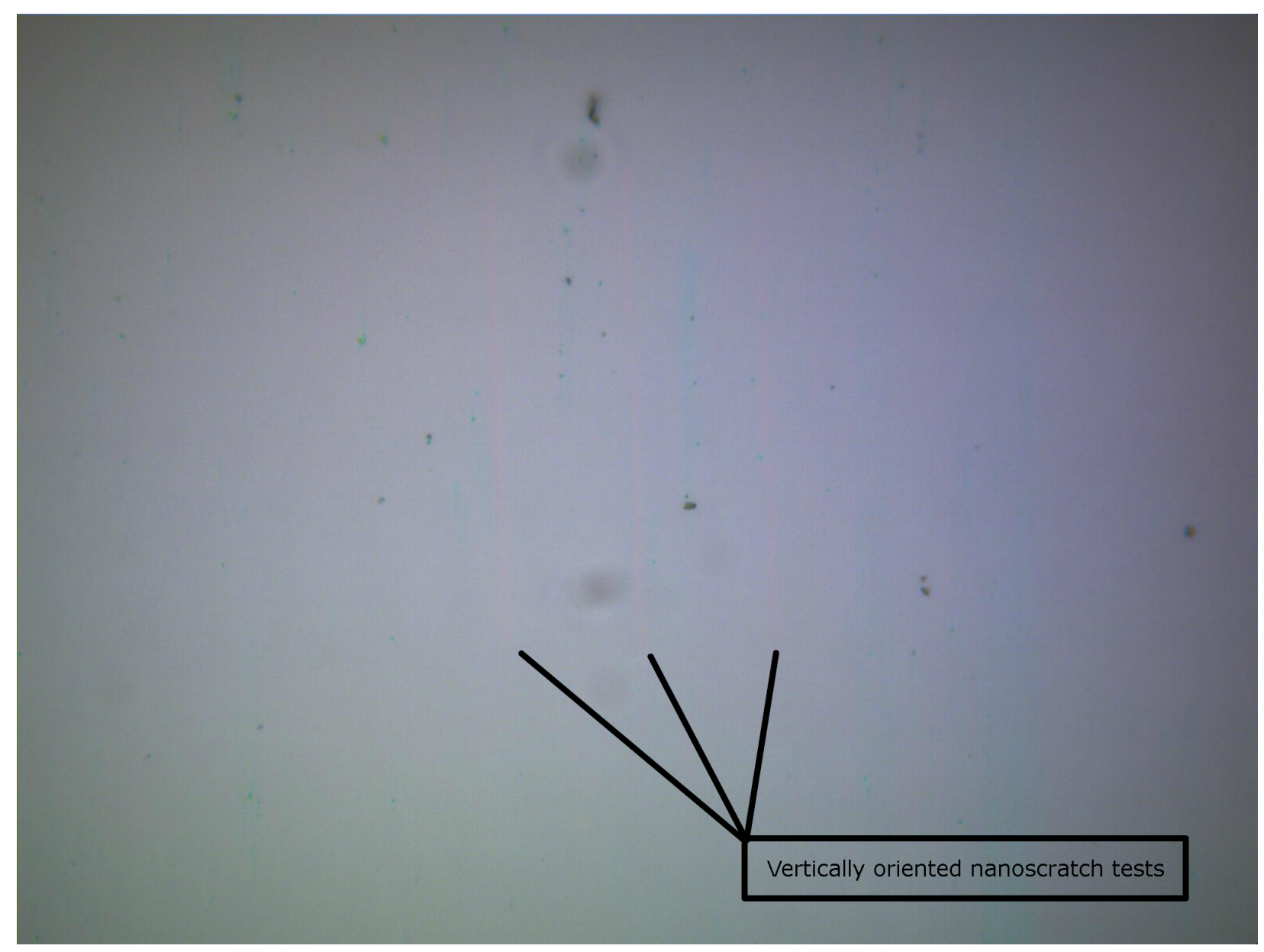

Figure 4.4: An example of a nanoscratch image. There are three vertical scratches in this image, parallel to each other and near the center of the field. They appear as redorange discolorations, just above each of the black label lines.

\subsection{Silicon Nitride Nanoindentation Results}

During the study, six samples from each of the three wafers underwent nanoindentation tests. These tests consisted of a series of 10 indents with identical indentation parameters. The parameters used in the indentation testing appear in Table 4.1 below: 
Table 4.1: Parameters used for nanoindentation testing.

\begin{tabular}{c|c}
\hline Available Load & $500 \mathrm{mN}$ \\
Applied Load & $50 \mathrm{mN}$ \\
Initial Load & $0.1 \mathrm{mN}$ \\
Loading Rate & $0.5 \mathrm{mN} / \mathrm{s}$ \\
$\begin{array}{c}\text { Unloading Rate } \\
\text { Dwell Period at maximum } \\
\text { load }\end{array}$ & $0.5 \mathrm{mN} / \mathrm{s}$ \\
Indentation Z offset & $60 \mathrm{~s}$ \\
Indentation Y offset & $20 \mu \mathrm{m}$ \\
\hline
\end{tabular}

\subsubsection{Nanoindentation Data Analysis Rationale}

The Nanotest analysis platform allows user control over the parameters used in the pyramidal analysis process, which converts the experiment data into useable results data. For the nanoindentation tests performed in this study, all pyramidal analysis was conducted using the parameters listed in the table below: 
Table 4.2: Pyramidal analysis parameters for analysis of nanoindentation data.

\begin{tabular}{|c|c|}
\hline Analysis Method & Power Law Fit \\
\hline Start curve fitting at: & $\begin{array}{c}100 \% \text { of maximum } \\
\text { load }\end{array}$ \\
\hline Stop curve fitting at: & $20 \%$ of maximum load \\
\hline Epsilon constant & 0.75 \\
\hline Frame compliance & 0.360933 \\
\hline $\begin{array}{c}\text { Thermal Drift } \\
\text { correction }\end{array}$ & $\begin{array}{l}\text { Use post-indentation } \\
\text { data }\end{array}$ \\
\hline
\end{tabular}

Moreover, the data gathered is dependent on the dimensions of the indenter tip used for data collection. The choice of using an ideal or general analysis function for the data analysis is also given to the user. The parameters used with the analysis function appear in the table below:

Table 4.3: Analysis function parameters for analysis of nanoindentation data.

\begin{tabular}{c|c}
\hline Function & General function \\
Beta factor & Berkovich; \\
& 1.03400 \\
$\begin{array}{c}\text { General } \\
\text { function }\end{array}$ & Fitted APd+BPD ${ }^{2}$ \\
\hline
\end{tabular}

As previously discussed, the nanoindentation analysis makes use of the equations derived by Oliver and Pharr that govern the behavior of a material compressed under indentation. 
The indentations in this study were carried out using a constant load during the indentation period, followed by a dwell period at the maximum planned indentation depth. The same constant load was applied during this dwell period, resulting in creep during this phase. The creep resulted in elastic deformation, causing an increase in the apparent indentation depth. Following the dwell period, the sample was unloaded. It is the curve recorded during the unloading process that is used to calculate the reduced elastic modulus, as the recovery of the sample is measured. This reduced modulus can then be used to find the actual sample elastic modulus, via the equation demonstrated by Oliver and Pharr. The area of the indenter tip is known: in confluence with the known load applied to the sample, the sample hardness can be simultaneously measured. Together, these allow for useful characterization of the sample surface through nanoindentation.

\subsubsection{Nanoindentation Elastic Modulus Results}

For each indent carried out, the reduced elastic modulus value reported by the Nanotest was recorded. The 10 data points generated in this way for each test series were then averaged to create a single datum for the test series. The data from the 18 nanoindentation test series were the source for the following analysis. Using the equation provided by Oliver and Pharr, the reduced modulus values were converted an elastic modulus value. 


\subsubsection{Nanoindentation Elastic Modulus Statistical Analysis}

The nanoindentation elastic modulus results were evaluated for normality using a Shapiro-Wilk test (JMP Pro V. 12.1, Cary, NC). The use of the Shapiro-Wilk test to verify data set normality was a recommended precursor to ANOVA testing of the results (C. Arnold, personal communication, December $3^{\text {rd }}$, 2016). The results of that test are presented below:

Table 4.4: Results of the Shapiro-Wilk Test for nanoindentation elastic modulus.

\begin{tabular}{c|c}
\hline Mean & $117.49 \mathrm{GPa}$ \\
Standard Deviation & $2.02 \mathrm{GPa}$ \\
Upper 95\% Mean & $118.50 \mathrm{GPa}$ \\
Lower 95\% Mean & $116.48 \mathrm{GPa}$ \\
Shapiro-Wilk W Test & 0.978492 \\
\hline
\end{tabular}

A W test value of over 0.9 was taken to indicate normality in the data set. With the data set verified as normal, the results could be evaluated further.

To evaluate the consistency of the elastic modulus of the silicon nitride thin films, an ANOVA (JMP Pro V. 12.1, Cary, NC) with elastic modulus as the continuous variable and wafer and quadrant as the covariates was run with a first-order interaction. Table 4.6 below displays the results of that ANOVA, where a p-value of less than 0.05 was taken to indicate a significant effect. 
Table 4.5: Results of the ANOVA for nanoindentation elastic modulus.

\begin{tabular}{cc} 
Effect & P \\
\hline Wafer & 0.2009 \\
Quadrant & 0.6980 \\
Wafer*Quadrant & 0.1921
\end{tabular}

The lack of significance for either of the main effects, as well as the lack of significance for the first-order interaction, demonstrates that there were no significant differences in the measured elastic modulus between either the quadrants of the wafers or between the wafers.

\subsubsection{Nanoindentation Hardness Results}

For each indent carried out, the hardness value reported by the Nanotest was recorded. The 10 data points generated in this way for each test series were then averaged to create a single datum for the test series. The data from the 18 nanoindentation test series were the source for the following analysis.

\subsubsection{Nanoindentation Hardness Statistical Analysis}

The nanoindentation hardness results were evaluated for normality using a Shapiro-Wilk test (JMP Pro V. 12.1, Cary, NC). The results of that test are presented below: 
Table 4.6: Results of the Shapiro-Wilk Test for nanoindentation hardness.

\begin{tabular}{c|c}
\hline Mean & $10.47 \mathrm{GPa}$ \\
Standard Deviation & $0.44 \mathrm{GPa}$ \\
Upper 95\% Mean & $10.69 \mathrm{GPa}$ \\
Lower 95\% Mean & $10.26 \mathrm{GPa}$ \\
Shapiro-Wilk W Test & 0.953450 \\
\hline
\end{tabular}

A W test value of over 0.9 was taken to indicate normality in the data set. With the data set verified as normal, the results could be evaluated further.

To evaluate the consistency of the hardness of the silicon nitride thin films, an ANOVA (JMP Pro V. 12.1, Cary, NC) with elastic modulus as the continuous variable and wafer and quadrant as the covariates was run with a first-order interaction. Table 4.8 below displays the results of that ANOVA, where a p-value of less than 0.05 was taken to indicate a significant effect.

Table 4.7: Results of the ANOVA for nanoindentation hardness.

\begin{tabular}{cc} 
Effect & P \\
\hline Wafer & 0.9184 \\
Quadrant & 0.2982 \\
Wafer*Quadrant & 0.7841
\end{tabular}

The lack of significance for either main effect, along with the lack of significance for the first-order interaction, indicates that there was no significant difference in the hardness of the silicon nitride coating either between the quadrants or between the wafers. 


\subsection{Silicon Nitride Nanoimpact Results}

During this study, 24 samples underwent nanoimpact testing. 12 samples were designated for sample oscillation testing. The parameters used in the sample oscillation testing are listed below.

Table 4.8: Sample oscillation test parameters

\begin{tabular}{c|c}
\hline Signal Generator Frequency & $20 \mathrm{~Hz}$ \\
Signal Generator Amplitude & $15 \mathrm{~V}$ \\
Limit Stop Load & $0.1 \mathrm{mN}$ \\
Impact Load & $5 \mathrm{mN}$ \\
Experiment Time & $300 \mathrm{~s}$ \\
\hline
\end{tabular}

The other 12 samples were used for pendulum oscillation testing. The parameters used in these tests are listed below:

Table 4.9: Pendulum oscillation test parameters

\begin{tabular}{c|c}
\hline Impulse Control On & $5 \mathrm{~s}$ \\
Impulse Control Off & $5 \mathrm{~s}$ \\
Limit Stop Load & $0.1 \mathrm{mN}$ \\
Impact Load & $5 \mathrm{mN}$ \\
Experiment Time & $600 \mathrm{~s}$ \\
\hline
\end{tabular}

\subsubsection{Nanoimpact Data Analysis Rationale}

The results from the nanoimpact tests are primarily concerned with the change in depth of the probe over the course of the testing. The change in depth, the time of the test, and the 
frequency of the impacts were used to generate a rate of change in depth per cycle. This rate was evaluated with the test type and the wafer and quadrant of the sample used for the test to search for meaningful differences.

\subsubsection{Nanoimpact Statistical Analysis}

The nanoimpact results were evaluated for normality by test type using a Shapiro-Wilk test (JMP Pro V. 12.1, Cary, NC). The results of that test for the sample oscillation results are presented below:

Table 4.10: Results of the Shapiro-Wilk Test for nanoimpact sample oscillation results.

\begin{tabular}{c|c}
\hline Mean & $0.1539 \mathrm{~nm} /$ cycle \\
Standard Deviation & $0.08113 \mathrm{~nm} /$ cycle \\
Upper 95\% Mean & $0.20840 \mathrm{~nm} /$ cycle \\
Lower 95\% Mean & $0.09939 \mathrm{~nm} /$ cycle \\
Shapiro-Wilk W Test & 0.836609 \\
\hline
\end{tabular}

A W test value of over 0.9 was taken to indicate normality in the data set. Because this data set was non-normal, the data set would need to be transformed in order to allow an ANOVA to be performed. To test for a lognormal distribution of the results, a Kolmogorov's D Test was performed on the data set (JMP Pro V. 12.1, Cary, NC). The results of that test for the sample oscillation results are presented below: 
Table 4.11: Results of the Kolmogorov's D Test for nanoimpact sample oscillation results.

\begin{tabular}{c|c}
\hline Mean & $0.1539 \mathrm{~nm} /$ cycle \\
Standard Deviation & $0.08113 \mathrm{~nm} /$ cycle \\
Upper 95\% Mean & $0.20840 \mathrm{~nm} /$ cycle \\
Lower 95\% Mean & $0.09939 \mathrm{~nm} /$ cycle \\
Kolmogorov's D Test & 0.216071 \\
\hline
\end{tabular}

In the Kolmogorov's D Test, a D test value of over 0.15 was taken to indicate a lognormal distribution in the data set. The sample oscillation results followed a lognormal distribution, but in order to transform the data set the pendulum oscillation results needed to be checked as well.

Table 4.12: Results of the Kolmogorov's D Test for nanoimpact pendulum oscillation results.

\begin{tabular}{c|c}
\hline Mean & $3.8653 \mathrm{~nm} /$ cycle \\
Standard Deviation & $2.2212 \mathrm{~nm} /$ cycle \\
Upper 95\% Mean & $5.2766 \mathrm{~nm} /$ cycle \\
Lower 95\% Mean & $2.4540 \mathrm{~nm} /$ cycle \\
Kolmogorov's D Test & 0.193704 \\
\hline
\end{tabular}

With the $\mathrm{D}$ test value for the pendulum oscillation results also above 0.15 , the data sets for both test types were confirmed to follow a lognormal distribution. To transform the data set, the natural logarithm of all of the depth change per cycle values was taken. In order to evaluate the normality of this data, another Shapiro-Wilk W test was performed on the transformed results of both test types. 
Table 4.13: Results of the Shapiro-Wilk $W$ test for the transformed sample oscillation data

\begin{tabular}{c|c}
\hline Mean & $-1.9854 \mathrm{~nm} /$ cycle \\
Standard Deviation & $0.4954 \mathrm{~nm} /$ cycle \\
Upper 95\% Mean & $-1.6525 \mathrm{~nm} /$ cycle \\
Lower 95\% Mean & $-2.3182 \mathrm{~nm} /$ cycle \\
Shapiro-Wilk W Test & 0.941772 \\
\hline
\end{tabular}

Table 4.14: Results of the Shapiro-Wilk W test for the transformed pendulum oscillation data

\begin{tabular}{c|c}
\hline Mean & $1.1698 \mathrm{~nm} /$ cycle \\
Standard Deviation & $0.6657 \mathrm{~nm} /$ cycle \\
Upper 95\% Mean & $1.5928 \mathrm{~nm} /$ cycle \\
Lower 95\% Mean & $0.7468 \mathrm{~nm} /$ cycle \\
Shapiro-Wilk W Test & 0.934093 \\
\hline
\end{tabular}

A Shapiro-Wilk W test value of 0.90 or greater was taken to indicate normality in the data set. With both transformed data sets verified as normal, the ANOVA could be performed.

To evaluate the resilience of the silicon nitride thin films to sample oscillation nanoimpact and pendulum oscillation nanoimpact, an ANOVA (JMP Pro V. 12.1, Cary, NC) with the natural logarithm of the change in depth per cycle as the continuous variable, and test type, wafer, and quadrant as the covariates, was run with a first-order interaction between wafer and quadrant. Table 4.15 below displays the results of that ANOVA, where a p-value of less than 0.05 was taken to indicate a significant effect. 
Table 4.15: Results of ANOVA for nanoimpact testing

\begin{tabular}{cc} 
Effect & P \\
\hline Wafer & 0.5935 \\
Quadrant & 0.6931 \\
Test Type & $<0.0001$ \\
Wafer*Quadrant & 0.8708
\end{tabular}

Significant differences for the rate of change of depth were found only for test type. The change in depth per cycle was significantly greater for the pendulum oscillation tests than for the sample oscillation tests. Figure 4.5 below displays the relative values for the rate of change of depth.

\section{Nanoimpact test type effect on the rate of change of depth}

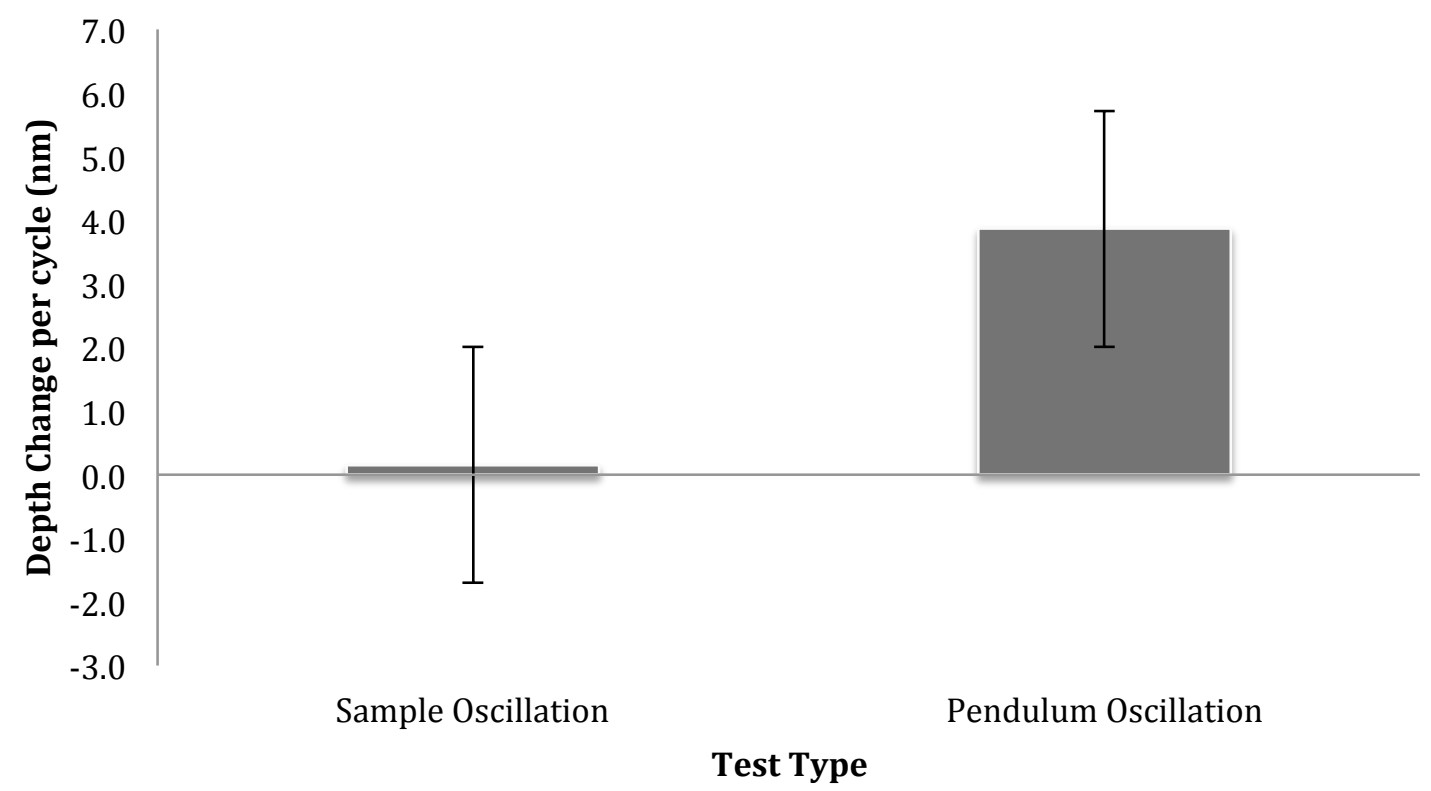

Figure 4.5: Test type effect on the rate of change of depth for silicon nitride thin films. 


\subsection{Silicon Nitride Nanoscratch Results}

During this study, 6 samples from each of the three wafers underwent scratch testing. The scratch testing consisted of performing a single Multi-Pass Wear test per sample with

varying parameters. The parameters used in the scratch tests are shown in Table 4.16 below:

Table 4.16: Parameters used in nanoscratch testing.

\begin{tabular}{ccc} 
Load $(\mathbf{m N})$ & Length $(\boldsymbol{\mu m})$ & Number of Included Tests \\
\hline 200 & 100 & 3 \\
150 & 149 & 4 \\
200 & 174 & 4 \\
200 & 200 & 5
\end{tabular}

The scratch lengths were specified to the NanoTest platform as $100 \mu \mathrm{m}, 150 \mu \mathrm{m}, 175 \mu \mathrm{m}$, and $200 \mu \mathrm{m}$. In practice, the scratch tests were carried out at the lengths listed in the above table instead. The reason for the discrepancy between the stipulated length and the actual length is unknown, but since the difference was consistent across tests the data was reported as it was collected.

\subsubsection{Nanoscratch Data Analysis Rationale}

The Nanotest platform reports the results of scratch testing primarily as a graph, relating the distance traveled by the probe through the pre-set scratch path with the depth recorded by the probe during the scratch. The maximum friction recorded by the friction transducer is presented within the same window. The scratch test data as reported is not 
subject to any further analysis within the NanoTest platform. Using the data reported by the NanoTest, the critical load of the coating can be found using the equations presented in Section 1.5.

\subsubsection{Nanoscratch Critical Load Statistical Analysis}

The nanoscratch critical load results were evaluated for normality using a Shapiro-Wilk test (JMP Pro V. 12.1, Cary, NC). The results of that test are presented below:

Table 4.17: Results of the Shapiro-Wilk Test for nanoscratch critical load.

\begin{tabular}{c|c}
\hline Mean & $289.38 \mathrm{mN}$ \\
Standard Deviation & $89.00 \mathrm{mN}$ \\
Upper 95\% Mean & $336.25 \mathrm{mN}$ \\
Lower 95\% Mean & $241.96 \mathrm{mN}$ \\
Shapiro-Wilk W Test & 0.923399 \\
\hline
\end{tabular}

A W test value of over 0.9 was taken to indicate normality in the data set. With the data set verified as normal, the results could be evaluated further.

To evaluate the consistency of the resilience of the silicon nitride thin films to scratching, an ANOVA (JMP Pro V. 12.1, Cary, NC) with critical load as the continuous variable and scratch load, scratch length, wafer, and quadrant as the covariates was run with firstorder interactions. Table 4.18 below displays the results of that ANOVA, where a p-value of less than 0.05 was taken to indicate a significant effect. 
Table 4.18: Results of a combined ANOVA for critical load.

\begin{tabular}{cc} 
Effect & P \\
\hline Wafer & 0.0099 \\
Quadrant (Test) & $<0.0001$ \\
Wafer*'Quadrant (Test) & 0.0191
\end{tabular}

Significant differences were found between the wafers, the quadrants (which were also the test types), and the interaction of the wafers and the test types. The results for the test types will be discussed first, in Figure 4.6 below:

\section{Quadrant (Test) effect on Critical Load}

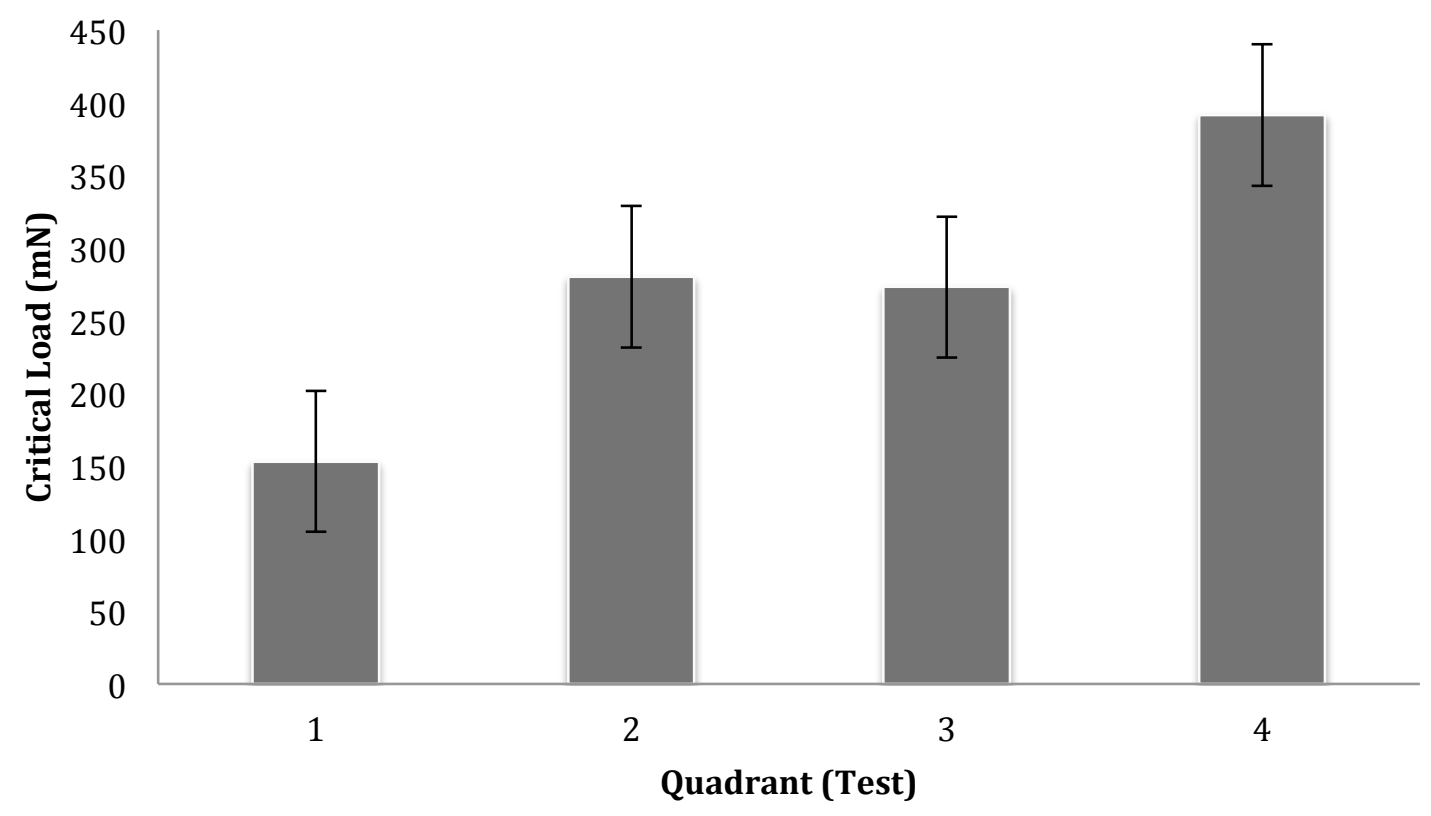

Figure 4.6: The effect of the Quadrant (Test) on the critical load. 
The results indicate that the measured critical load is roughly directly proportional to the test parameters, i.e. the load and the length of the scratch. These inform the work done during the scratch. This effect was shown as highly significant in the final results.

The wafer used for the test was also shown as a significant effect on the critical load. A graphical comparison of the wafer effects is shown as Figure 4.7:

\section{Wafer effect on Critical Load}

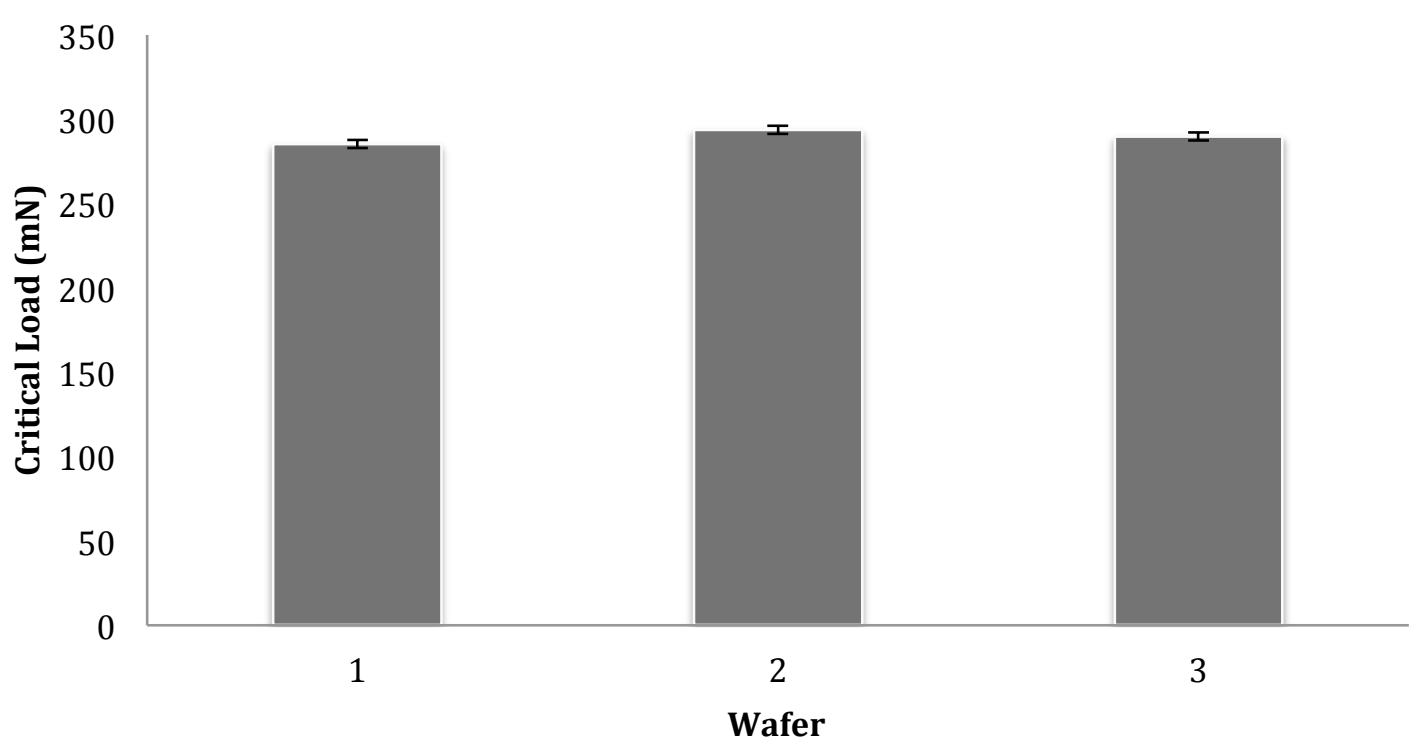

Figure 4.7: The effect of the wafer on the critical load.

The wafers all had exceptionally close mean reported critical load values. The implications of the limited sample size on the result will be discussed in Section 5.0 


\subsection{Discussion}

The nanoindentation testing of the silicon nitride thin films shows the consistency of the hardness and elastic modulus of those films. Across hundreds of individual indentations made, no significant difference in the values of those properties was demonstrated between quadrants on the same wafer, between different wafers, or even between specific quadrants on the different wafers. From this, it can be concluded that the fabrication techniques used to produce the films creates a highly consistent result.

A reliable method to evaluate the hardness and elastic modulus of a thin coating over a substrate is of substantial and continuing interest. Recent research in the field of thin-film ceramic coatings has favored coatings that feature hard yet tough coatings [27-30]. Silicon nitride is a strong candidate for these roles in biomedical engineering due to its favorable biocompatibility properties $[12,16]$. The same nanoindentation techniques used in this study can be applied to efficiently characterize coatings produced for these applications.

The nanoimpact testing conducted in this study demonstrated that the results in the rate of damage to the silicon nitride coating were dependent on the test type selected. The damage to the surface per cycle was significantly higher for the pendulum oscillation technique than for the sample oscillation technique. The two methods were both used with $5 \mathrm{mN}$ of force applied to the surface. Although the frequency of impact and the total time of the test were different between the methods, recording the results as the change in depth per impact cycle removed the influence of those differences on the data set. 
Nanoimpact testing is less popular than the similar but better-established nanoindentation technique, it has been used as a characterization method for deposited thin films [31]. The characterization of thin films via nanoimpact offers an opportunity to examine the effect of the coating-substrate interaction [32-34], which will be of substantial interest in any industrial application of ceramic thin films. This study indicated that the method chosen for the nanoimpact testing could have an effect on the results, even when there were no meaningful differences between the samples used.

The nanoscratch testing conducted in this study demonstrated that the critical load of the coating was influenced both by the type of test conducted on the sample as well as by the wafer of origin of the sample. This data set was limited in scope, primarily due to difficulties with the nanoscratch apparatus. As a result, any conclusions drawn from this study must be weighed against the small sample size being used. It has previously been demonstrated that the measured critical load from a nanoscratch test can be affected by the test parameters [35]. This corresponds to the findings of this study and suggests that use of the nanoscratch test to characterize a coating should be restricted to a uniform set of testing parameters in order to minimize variation. On the other hand, the limited sample size may be exaggerating the significance of effects in the final model. These results bear further investigation, but they should not be considered authoritative in the format that they are presented in this study.

Results obtained by this study could have been adversely affected by environmental factors acting on the testing apparatus. Micro- and nanoscale testing operations are by 
nature very delicate procedures, and variations in temperature and vibration could have affected data gathered by this study. As discussed in Section 3, the NanoTest apparatus used for data collection was stored on a vibration-isolating table within a temperaturecontrolled cabinet; this cabinet was itself stored within a temperature-controlled room. These were judged sufficient controls to mitigate the chance of environmental distortion of the data, or at least distribute any distortion sources equally. Despite these efforts, some tests returned unusable results. No precise cause for the distortion of the data can be assigned, so any failed data sets were excluded from analysis in the final report.

The outcomes of the study allow each hypothesis proposed in Section 2 to be evaluated.

Hypothesis 1: The silicon nitride thin films will have highly consistent hardness and elastic modulus as measured by nanoindentation, both between wafer quadrants and between wafers. The nature of the fabrication process for the nitride thin films should prevent significant deviation. The collected data support this hypothesis. There was not a statistically significant effect observed on either the hardness or the elastic modulus when taking into account the wafer and the quadrant used for testing, as well as the first-order interaction between the groups.

Hypothesis 2: The silicon nitride thin films will be more resistant to fracture from pendulum oscillation than from sample oscillation, as measured by the damage rate experienced by the coating due to each test type. Although the initial force experienced by the sample is identical in both cases, the higher frequency of the sample oscillation 
technique will result in damage to the film structure accumulating at a higher rate. The collected data do not support this hypothesis. The rate of damage under the pendulum oscillation test type was significantly higher than that observed for the sample oscillation test type. Since the hardness and elastic modulus of the samples were established to not vary significantly during the testing of Hypothesis 1 , and the load applied via the indentation techniques was identical, the frequency and total number of impacts cannot have an effect on the damage rate. The mechanism by which the greater rate of damage from pendulum oscillation is occurring will require further study.

Hypothesis 3: The critical load of the silicon nitride thin film on its' substrate will be consistent between all test parameters. Changing the testing parameters should not create inconsistencies with the film properties. The results do not support this hypothesis. The wafer of origin of the sample appeared to be a significant effect, as did the interaction between the wafer of origin and the test type employed. The small sample size involved in the model means that these conclusions cannot be considered authoritative. Further study would be required to establish how accurate the significance of all three effects is to the critical load of the coating. 


\subsection{Conclusions and Recommendations}

The hardness and elastic modulus of silicon nitride thin films deposited onto wafers appears to be highly consistent across samples. Moreover, the technique of nanoindentation appears well suited to evaluate these properties in an efficient way. While this study evaluated only one particular film configuration, the test procedure was validated.

The damage to a thin film from pendulum oscillation nanoimpacts appears to be significantly greater than those from sample oscillation nanoimpacts. The effect of the impact frequency on coating degradation is something that should be investigated in a

future study, perhaps concurrently with nanoindentation studies of thin films on substrates. By using the same loading condition on a single coating with only one of the nanoimpact tests, any correlation between the frequency of impact and the damage rate could be characterized successfully. Understanding the limitations of silicon nitride or other thin films in real-world applications would benefit from this effect being explored further.

The sample oscillation nanoindentation technique has one significant disadvantage. The technique requires a piezoelectric sample stage, a unique item that can be damaged with the application of torque. The pendulum oscillation technique is more robust and requires less care and fewer additional system components to perform. 
A disadvantage of the nanoimpact testing technique as a whole is the creation of a debris field in the immediate vicinity of the impacts. While there is no evidence to suggest that this has an effect on the results of the testing, the debris field can contaminate a relatively wide area of a sample surface. On samples that do not have a large area for testing to begin with, this could be a significant handicap.

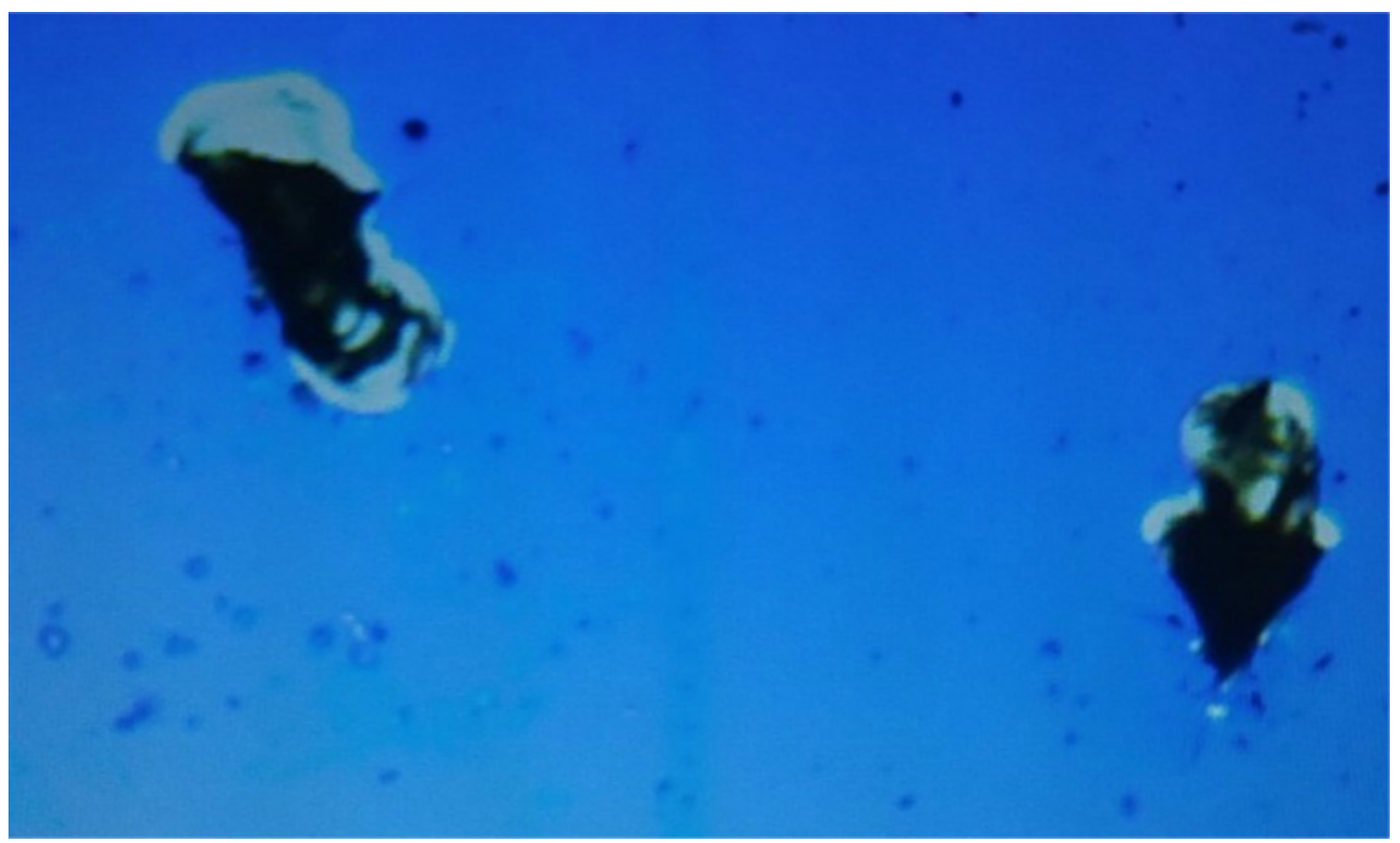

Figure 6.1: An example of a nanoimpact test site with a significant debris field. Note that the shape of the cube corner indenter is distorted. 
The nanoscratch results indicate that the test parameters in conjunction with the wafer of origin effect the critical load of the silicon nitride coating. This conclusion should be reevaluated with a larger sample size before being understood as definitive. In future work, understanding the delamination conditions for a coating that is designed to resist wear and protect an underlying substrate is critically important. The nanoscratch technique is vulnerable to the fragility of the system and the low efficiency of data collection compared to the nanoindentation and nanoimpact techniques, but remains valuable for the unique results that it can provide with a proper sample cohort.

Meaningful future work can be carried out to expand upon the techniques evaluated here. Any biomedical or orthopedic application of a silicon nitride thin film, or any other wearresistant film, would most likely use an existing biomedical material as its substrate rather than single-crystal silicon. Examples of useful substrates could include stainless steel, or preferably titanium alloys or chromium-molybdenum steel.

Future work with the NanoTest device would benefit from scheduling tests to occur exclusively at night. Most of the failed data points collected during this study were collected during daytime, when the lab had unrestricted activity. Tests conducted at night, when the lab was empty, generally had fewer failed points. This is likely the result of a more consistent environment, without random vibrations an fluctuations in temperature that are present in a busy lab. 


\section{REFERENCES}

[1] Bal, B Sonny, and MN Rahaman. (2012): "Orthopedic applications of silicon nitride ceramics." Acta biomaterialia 8.8 2889-2898

[2] McEntire, BJ et al. (2015): "Ceramics and ceramic coatings in orthopaedics." Journal of the European Ceramic Society 35.16 4327-4369.

[3] Becher, Paul F et al. "Microstructural design of silicon nitride with improved fracture toughness: I, effects of grain shape and size." Journal of the American Ceramic Society-Including Communications of the American Ceramic Society 81.11 (1998): 2821-2830.

[4] Freiman, SW, DR Mulville, and P Wno Mast. "Crack propagation studies in brittle materials." Journal of Materials Science 8.11 (1973): 1527-1533.

[5] Filsinger, D et al. "Model combustor to assess the oxidation behavior of ceramic materials under real engine conditions." ASME 1999 International Gas Turbine and Aeroengine Congress and Exhibition 7 Jun. 1999: V004T02A013-V004T02A013.

[6] Li, Da et al. "Silicon nitride and intrinsic amorphous silicon double antireflection coatings for thin-film solar cells on foreign substrates." Thin Solid Films 583 (2015): 25-33.

[7] Cheng, Zaijun et al. "Effect of arsenic doping on charge relaxation process in silicon nitride film for capacitive RF MEMS switch application." Microelectronic Engineering 162 (2016): 89-92.

[8] Wu, Cheng-Yu, Chun-Chi Chang, and Jenq-Gong Duh. "Silicon nitride coated silicon thin film on three dimensions current collector for lithium ion battery anode." Journal of Power Sources 325 (2016): 64-70.

[9] Dalal, Ali et al. (2012): "Orthopedic implant cobalt alloy particles produce greater toxicity and inflammatory cytokines than titanium alloy and zirconium alloy based particles in vitro, in human osteoblasts, fibroblasts, and macrophages." Journal of Biomedical Materials Research Part A 100.8 2147-2158.

[10] Waris, Ville et al. (2012): "Role and regulation of VEGF and its receptors 1 and 2 in the aseptic loosening of total hip implants." Journal of Orthopaedic Research 30.11 $1830-1836$.

[11] Samelko, Lauryn et al. (2013): "Cobalt-alloy implant debris induce HIF-1 $\alpha$ hypoxia associated responses: A mechanism for metal-specific orthopedic implant failure." PloS one 8.6 e67127. 
[12] Neumann, A et al. (2004): "Comparative investigation of the biocompatibility of various silicon nitride ceramic qualities in vitro." Journal of Materials Science: Materials in Medicine 15.10 1135-1140.

[13] Kue, R et al. (1999): "Enhanced proliferation and osteocalcin production by human osteoblast-like MG63 cells on silicon nitride ceramic discs." Biomaterials 20.13 1195-1201.

[14] Pettersson, Maria et al. (2013): "Mechanical and tribological behavior of silicon nitride and silicon carbon nitride coatings for total joint replacements." Journal of the mechanical behavior of biomedical materials 25 41-47.

[15] Guedes e Silva, Cecilia C et al. (2008): "Tissue response around silicon nitride implants in rabbits." Journal of Biomedical Materials Research Part A 84.2 337343.

[16] Webster, Thomas J et al. (2012): "Anti-infective and osteointegration properties of silicon nitride, poly (ether ether ketone), and titanium implants." Acta biomaterialia $8.124447-4454$.

[17] Shi, Zhifeng et al. (2012): "Silicon nitride films for the protective functional coating: Blood compatibility and biomechanical property study." journal of the mechanical behavior of biomedical materials 16 9-20.

[18] Oliver, W.C. and Pharr, G.M. (1992): “An Improved Technique for Determining Hardness and Elastic Modulus Using Load and Displacement Sensing Indentation Experiments." Journal of Materials Research 7.6 1564-1583.

[19] Doerner, M.F. and Nix, W.D. (1986) "A method for interpreting the data from depth-sensing indentation instruments." Journal of Materials Research 1.4 601-609.

[20] Hainsworth, S.V. et al. (1996): "Analysis of nanoindentation load-displacement loading curves.” Journal of Materials Research 11.8 1987-1995.

[21] Oliver, W.C. and Pharr, G.M. (2004) "Measurement of hardness and elastic modulus by instrumented indentation: Advances in understanding and refinements to methodology." Journal of Materials Research 19 3-20.

[22] Laugier, Michael T. (1984) "An energy approach to the adhesion of coatings using the scratch test." Thin Solid Films 117.4 243-249.

[23] Burnett, PJ, and DS Rickerby. (1987) "The relationship between hardness and scratch adhesion." Thin solid films 154.1-2 403-416. 
[24] Attar, Fouad, and Thomas Johannesson. (1996) "Adhesion evaluation of thin ceramic coatings on tool steel using the scratch testing technique." Surface and Coatings Technology 78.1 87-102.

[25] Bull, SJ et al. (1988) "The use of scratch adhesion testing for the determination of interfacial adhesion: the importance of frictional drag." Surface and Coatings technology 36.1-2 503-517.

[26] Lei, Weisheng et al. (2012) "Die singulation technologies for advanced packaging: a critical review." Journal of Vacuum Science \& Technology B Microelectronics and Nanometer Structures 30.4 1-27.

[27] D.G. Sangiovanni et al. (2011) "Supertoughening in B1 transition metal nitride alloys by increased valence electron concentration" Acta Materialia 59 2121-2134

[28] Y.X. Wang et al. (2014) "Toward hard yet tough ceramic coatings" Surface Coatings Technology 258 1-16

[29] J. Musil (2015) "Advanced hard coatings with enhanced toughness and resistance to cracking" Thin Films and Coatings: Toughening and Toughness Characterisation 7 $378-463$

[30] X. Zhang et al. (2015) "Toughness evaluation of thin hard coatings and films" Thin Films and Coatings: Toughening and Toughness Characterisation 2 48-121

[31] N. Faisal et al. (2012) "Nano-impact testing and failure mechanism of solar panel DLC film” Surface and Coatings Technology 2 195-209

[32] Chen, Jian et al. (2016) "Load sensitivity in repetitive nano-impact testing of TiN and AlTiN coatings" Surface and Coatings Technology 308.25 289-297

[33] Galvan, D et al. (2006) "Deformation and failure mechanism of nano-composite coatings under nano-indentation”. Surface and Coatings Technology 200.24 67186726

[34] Pei, Y.T. et al. (2005) "Nanostructure and properties of TiC/a-C:H composite coatings" Acta Materialia 53.17 4505-4521

[35] Wang, X., Yoon, Y. J., \& Ji, H. (2007: “A novel scratching approach for measuring age-related changes in the in situ toughness of bone". Journal of Biomechanics, 40(6), 1401-1404. 


\section{APPENDIX: DATA STATISTICS}

\section{Nanoindentation Elastic Modulus Data: Distribution of Results}

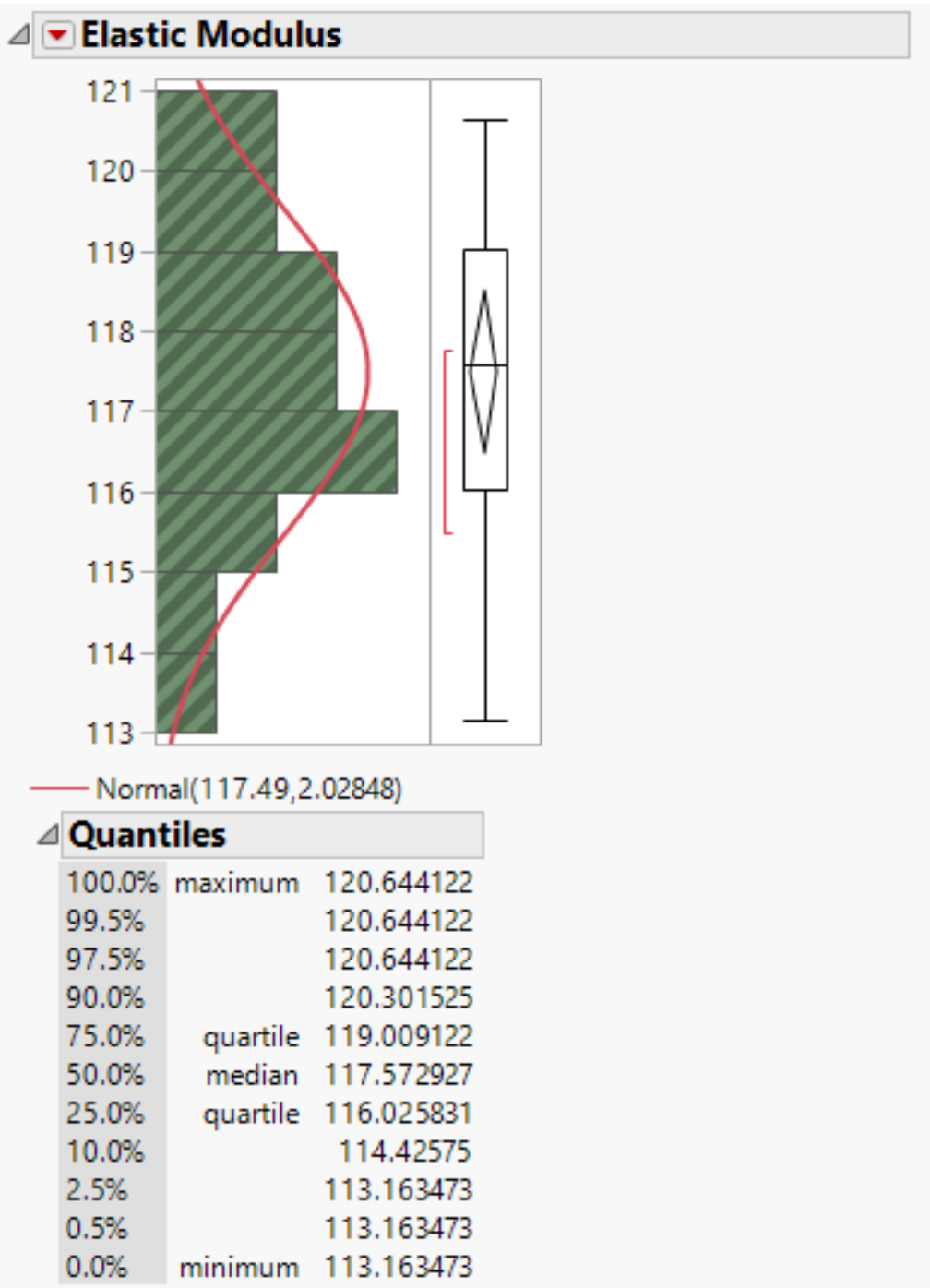




\begin{tabular}{|c|c|c|c|c|}
\hline \multicolumn{2}{|c|}{$\Delta \nabla$ Summary Statistics } & & & \\
\hline Mean & \multicolumn{4}{|c|}{117.49003} \\
\hline Std Dev & \multicolumn{4}{|c|}{2.0284796} \\
\hline Std Err Mean & \multicolumn{4}{|c|}{0.4781172} \\
\hline Upper $95 \%$ Mean & \multicolumn{4}{|c|}{ In 118.49877} \\
\hline Lower $95 \%$ Mean & \multicolumn{4}{|c|}{ in 116.4813} \\
\hline N & \multicolumn{4}{|c|}{18} \\
\hline \multicolumn{5}{|c|}{$\Delta \sim$ Fitted Normal } \\
\hline \multicolumn{5}{|c|}{$\triangle$ Parameter Estimates } \\
\hline Type & Parameter & Estimate & Lower $95 \%$ & Upper $95 \%$ \\
\hline Location $\mu$ & & 117.49003 & 116.4813 & 118.49877 \\
\hline Dispersion $\sigma$ & & 2.0284796 & 1.5221459 & 3.040983 \\
\hline \multicolumn{5}{|c|}{$-2 \log ($ Likelihood $)=75.5441030159375$} \\
\hline \multicolumn{5}{|c|}{$\Delta$ Goodness-of-Fit Test } \\
\hline \multicolumn{5}{|c|}{ Shapiro-Wilk W Test } \\
\hline W & \multicolumn{4}{|c|}{ Prob $<W$} \\
\hline 0.978492 & \multicolumn{4}{|c|}{0.9331} \\
\hline \multicolumn{5}{|c|}{$\begin{array}{l}\text { Note: } \mathrm{Ho}=\text { The data is from the Normal distribution. Small p- } \\
\text { values reject Ho. }\end{array}$} \\
\hline
\end{tabular}


Nanoindentation Elastic Modulus Data: Full ANOVA Results

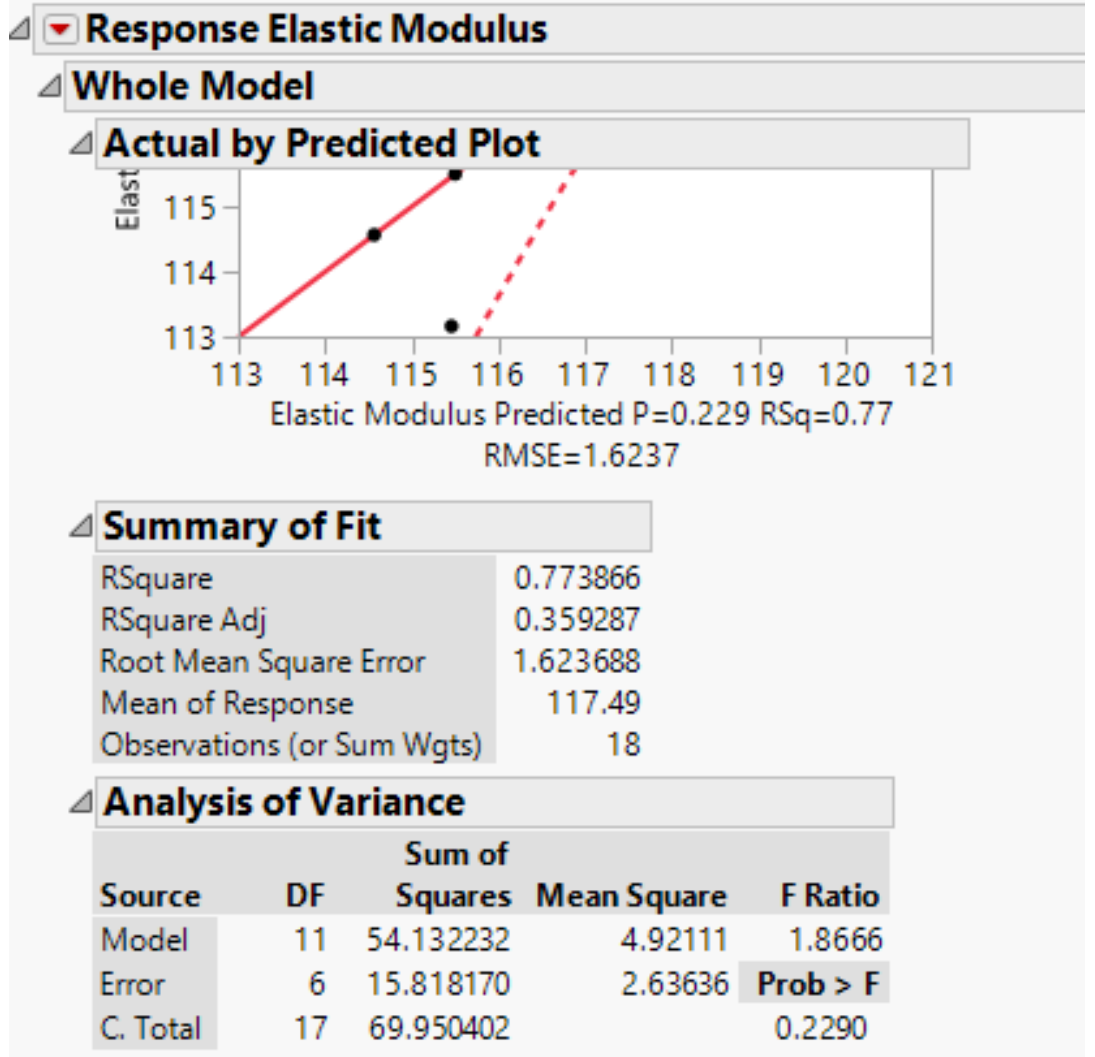

\section{$\triangleright$ Parameter Estimates}

\section{$\triangle$ Effect Tests}

\begin{tabular}{lrrrrrr} 
& \multicolumn{5}{c}{ Sum of } \\
Source & Nparm & DF & Squares & F Ratio & Prob > F \\
Wafer & 2 & 2 & 11.189180 & 2.1221 & 0.2009 \\
Quadrant & 3 & 3 & 3.926871 & 0.4965 & 0.6980 \\
Wafer*Q Quadrant & 6 & 6 & 33.438738 & 2.1139 & 0.1921
\end{tabular}

\section{$\triangle$ Residual by Predicted Plot}

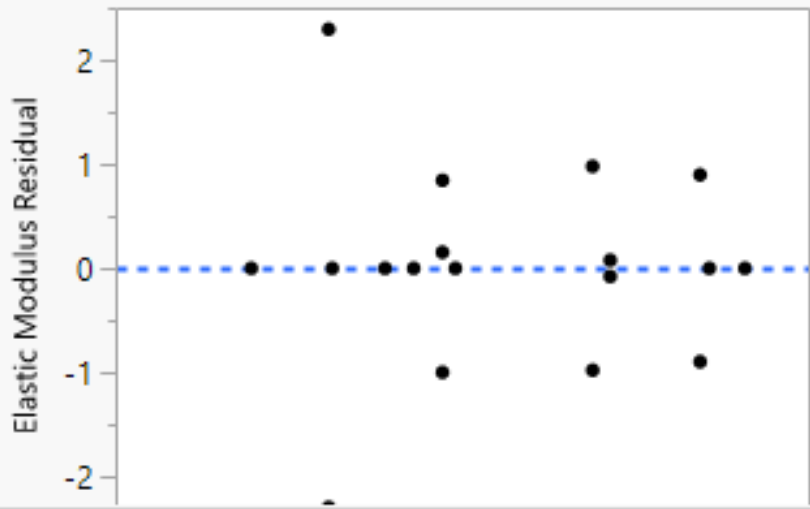




\begin{tabular}{|c|c|c|c|c|c|}
\hline \multicolumn{6}{|c|}{$\triangle$ Parameter Estimates } \\
\hline Term & \multicolumn{2}{|c|}{ Estimate } & Std Error & t Ratio & Prob $>|t|$ \\
\hline Intercept & \multicolumn{2}{|c|}{117.40434} & 0.413371 & 284.02 & $<.0001^{*}$ \\
\hline Wafer[1] & \multicolumn{2}{|c|}{-1.198239} & 0.594942 & -2.01 & 0.0906 \\
\hline Wafer[2] & \multicolumn{2}{|c|}{0.8145673} & 0.579351 & 1.41 & 0.2093 \\
\hline Quadrant[1] & \multicolumn{2}{|c|}{-0.203505} & 0.715979 & -0.28 & 0.7858 \\
\hline Quadrant[2] & \multicolumn{2}{|c|}{-0.360459} & 0.732828 & -0.49 & 0.6403 \\
\hline Quadrant[3] & \multicolumn{2}{|c|}{0.8273672} & 0.681032 & 1.21 & 0.2700 \\
\hline Wafer[1] $]^{\star}$ Quadrant[1] & \multicolumn{2}{|c|}{0.7704546} & 0.917699 & 0.84 & 0.4333 \\
\hline Wafer[1] $]^{\star}$ Quadrant[2] & \multicolumn{2}{|c|}{-0.345066} & 1.076806 & -0.32 & 0.7595 \\
\hline Wafer[1] $]^{\star}$ Quadrant[3] & \multicolumn{2}{|c|}{-0.592461} & 1.042247 & -0.57 & 0.5904 \\
\hline Wafer[2]*Quadrant[1] & \multicolumn{2}{|c|}{-3.449403} & 1.056784 & -3.26 & $0.0172^{*}$ \\
\hline Wafer[2]*Quadrant[2] & \multicolumn{2}{|c|}{0.8504413} & 0.959951 & 0.89 & 0.4098 \\
\hline Wafer[2]*Quadrant[3] & \multicolumn{2}{|c|}{0.7007986} & 0.921018 & 0.76 & 0.4755 \\
\hline \multicolumn{6}{|l|}{$\triangle$ Effect Tests } \\
\hline Source & \multicolumn{2}{|c|}{ Nparm } & $\begin{array}{r}\text { Sum of } \\
\text { Squares }\end{array}$ & F Ratio & - Prob $>F$ \\
\hline Wafer & 2 & 2 & 11.189180 & 2.1221 & $1 \quad 0.2009$ \\
\hline Quadrant & 3 & 3 & 3.926871 & 0.4965 & $5 \quad 0.6980$ \\
\hline Wafer*Quadrant & 6 & 6 & 33.438738 & 2.1139 & $9 \quad 0.1921$ \\
\hline
\end{tabular}

\section{$\triangle$ Residual by Predicted Plot}

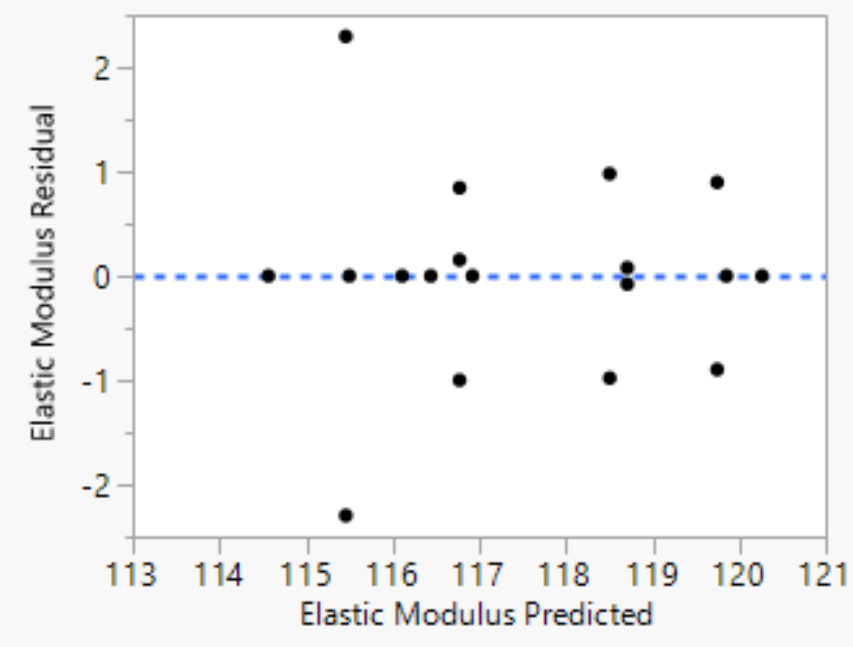




\section{Nanoindentation Hardness Data: Distribution of Results}

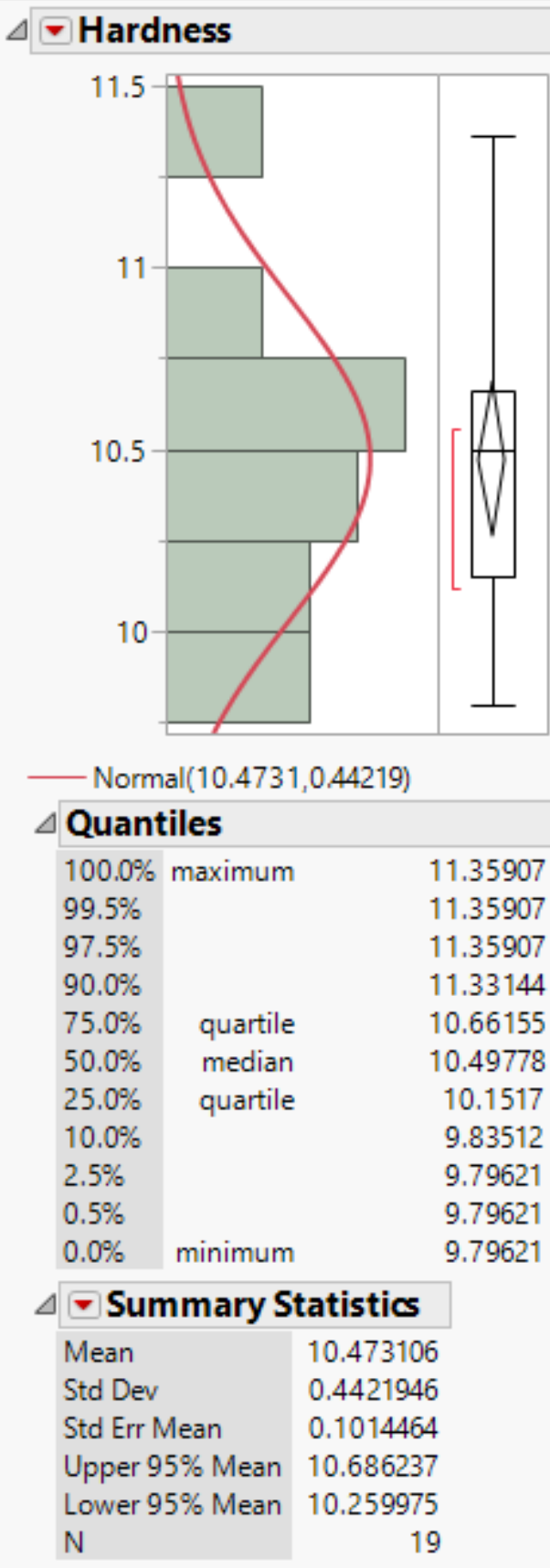




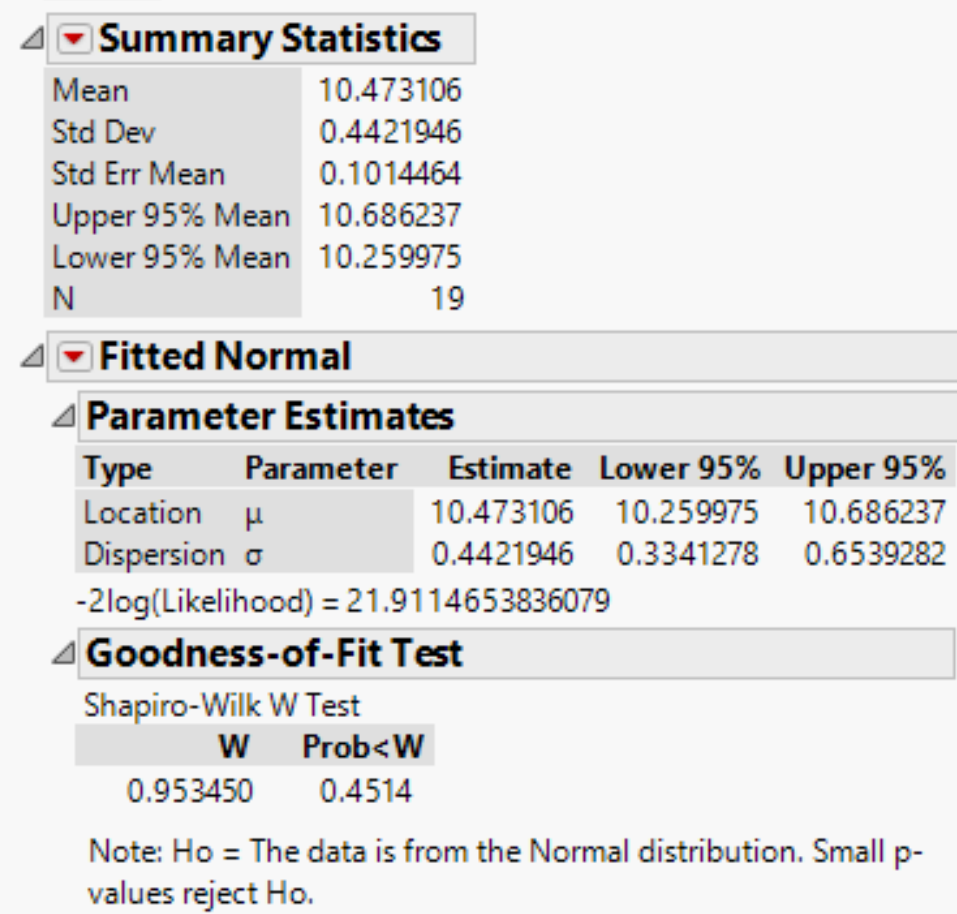




\section{Nanoindentation Hardness Data: Full ANOVA Results}

$\Delta$ Response Hardness
$\Delta$ Whole Model
$\Delta$ Effect Summary

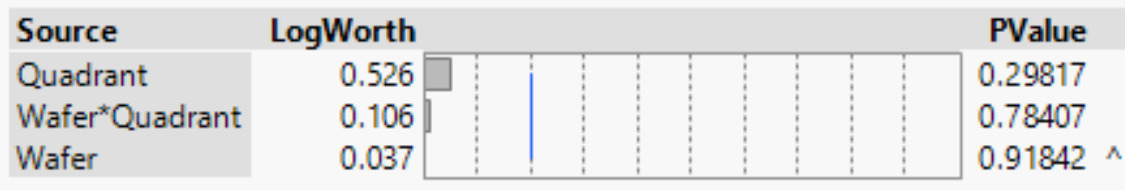

Remove Add Edit $\square$ FDR (" $\wedge$ ' denotes effects with containing effects above them)

\section{$\triangle$ Actual by Predicted Plot}

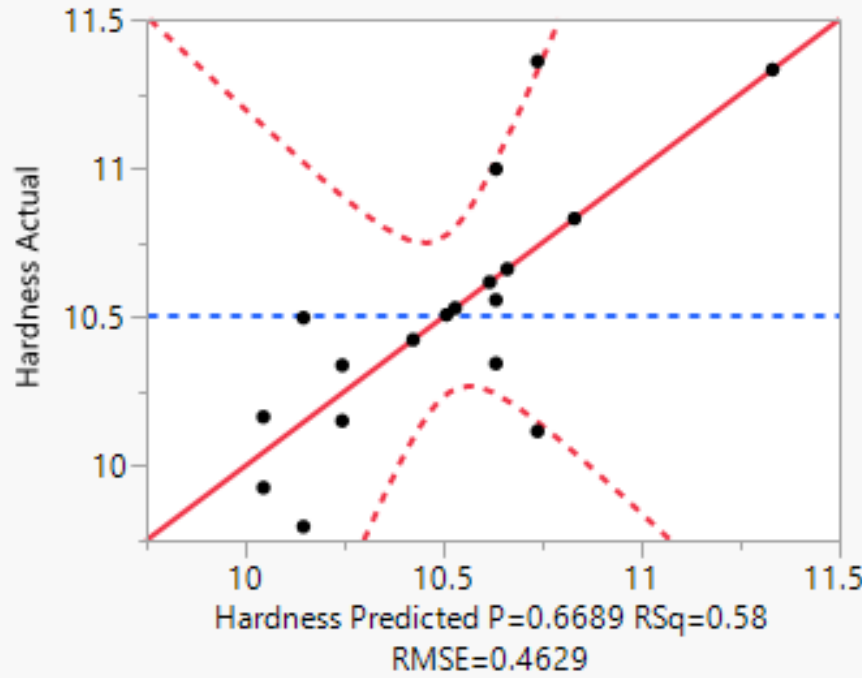

\section{$\triangle$ Summary of Fit}

\begin{tabular}{l|r}
\hline RSquare & 0.583898 \\
\hline RSquare Adj & -0.17896 \\
\hline Root Mean Square Error & 0.462918 \\
\hline Mean of Response & 10.50855 \\
\hline Observations (or Sum Wgts) & 18
\end{tabular}

$\triangle$ Analysis of Variance

\begin{tabular}{|c|c|c|c|c|}
\hline Source & DF & $\begin{array}{l}\text { Sum of } \\
\text { Squares }\end{array}$ & Mean Square & F Ratio \\
\hline Model & 11 & 1.8042508 & 0.164023 & 0.7654 \\
\hline Error & 6 & 1.2857598 & 0.214293 & Prob $>F$ \\
\hline C. Total & 17 & 3.0900107 & & 0.6689 \\
\hline
\end{tabular}




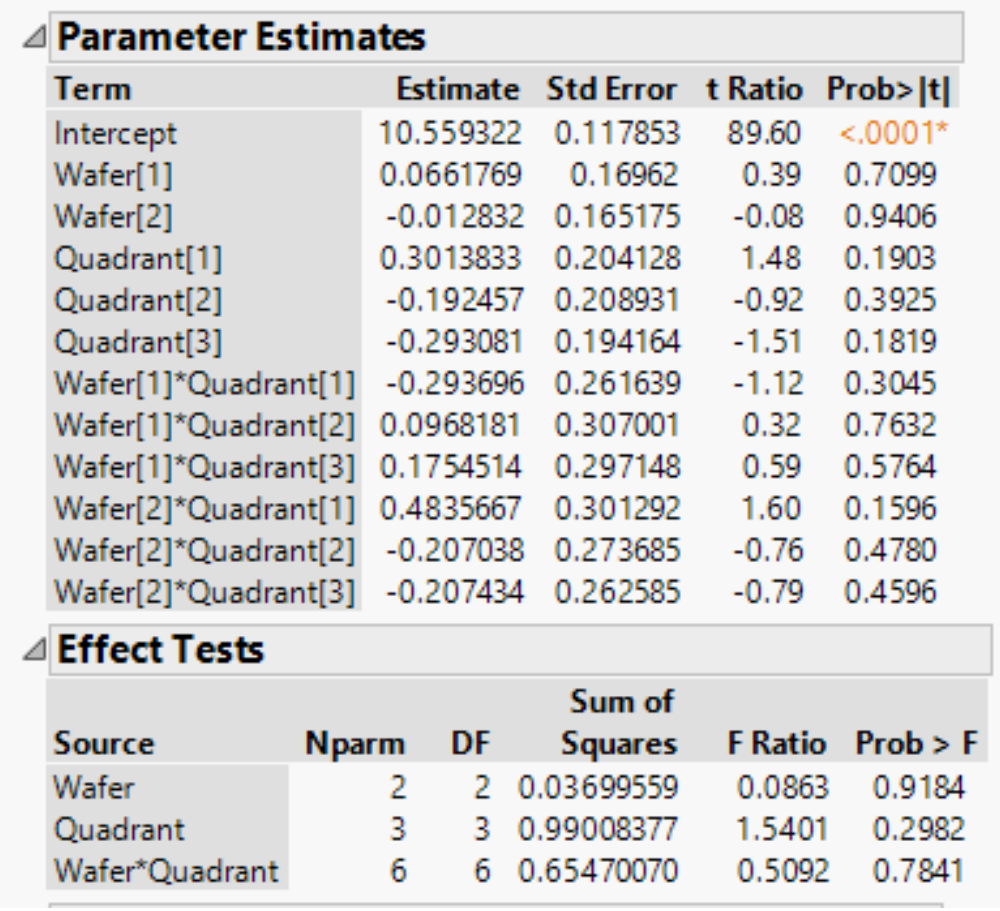


Nanoimpact Sample Oscillation Data: Distribution of Results

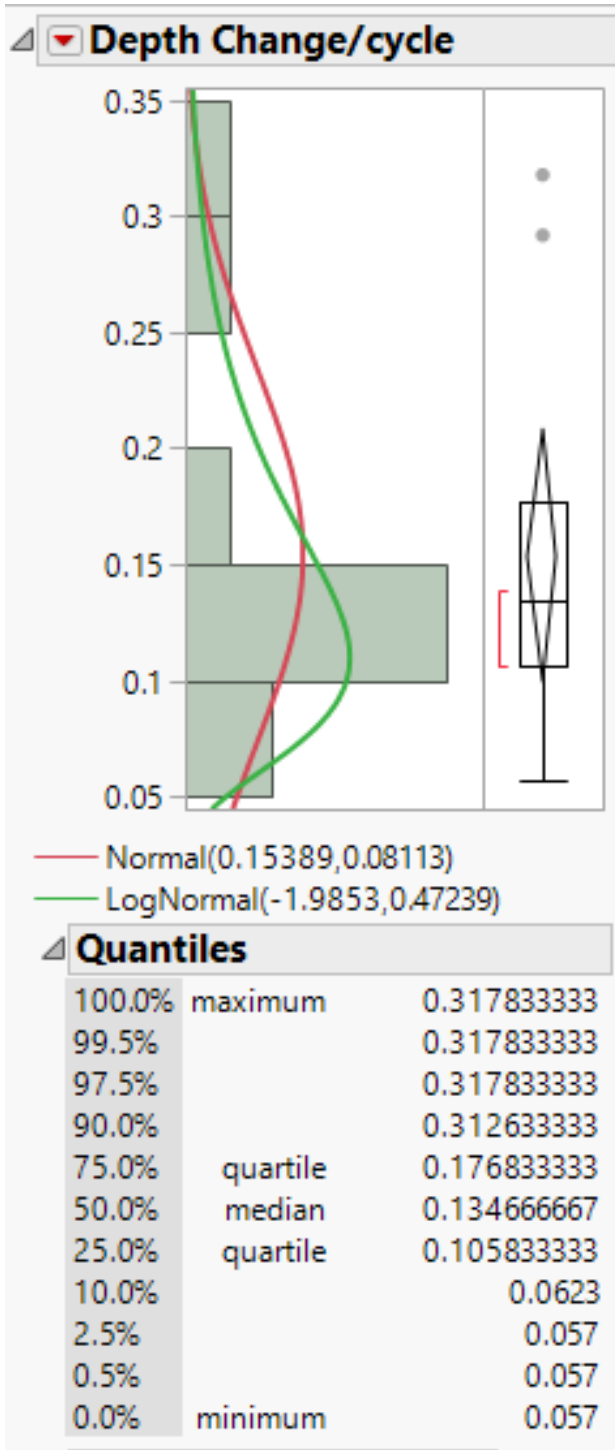




\begin{tabular}{|c|c|}
\hline \multicolumn{2}{|c|}{$\triangle \nabla$ Summary Statistics } \\
\hline Mean & 0.1538939 \\
\hline Std [ & 0.08 \\
\hline Std Err Mean & 0.0244607 \\
\hline Upper $95 \%$ Mean & 0.2083957 \\
\hline Lower $95 \%$ Mean & 0.0993921 \\
\hline $\mathrm{N}$ & 11 \\
\hline
\end{tabular}

\begin{tabular}{|c|c|c|c|c|}
\hline \multicolumn{5}{|c|}{$\Delta-$ Fitted Normal } \\
\hline \multicolumn{5}{|c|}{$\triangle$ Parameter Estimates } \\
\hline Type & Parameter & Estimate & Lower $95 \%$ & Upper $95 \%$ \\
\hline Location & $\mu$ & 0.1538939 & 0.0993921 & 0.2083957 \\
\hline Dispersion & $\sigma$ & 0.0811269 & 0.0566847 & 0.1423723 \\
\hline
\end{tabular}

\section{$\triangle$ Goodness-of-Fit Test}

Shapiro-Wilk W Test

W Prob $<W$

$0.8366090 .0285^{*}$

Note: Ho $=$ The data is from the Normal distribution. Small $p-$ values reject $\mathrm{Ho}$.

\begin{tabular}{|c|c|c|c|}
\hline \multicolumn{4}{|c|}{$\Delta-$ Fitted LogNormal } \\
\hline \multicolumn{4}{|c|}{$\triangle$ Parameter Estimates } \\
\hline Type Parameter & Estimate & Lower $95 \%$ & Upper $95 \%$ \\
\hline Scale $\mu$ & -1.985349 & -2.29075 & -1.679949 \\
\hline Shape $\sigma$ & 0.4723884 & 0.3273016 & 0.7673283 \\
\hline \multicolumn{4}{|c|}{$-2 \log ($ Likelihood $)=-28.9600180700951$} \\
\hline \multicolumn{4}{|c|}{$\Delta$ Goodness-of-Fit Test } \\
\hline \multicolumn{4}{|l|}{ Kolmogorov's D } \\
\hline D & \\
\hline $0.216071>$ & \multicolumn{3}{|c|}{$\begin{array}{c}\text { Prob }>\text { D } \\
0.1500\end{array}$} \\
\hline
\end{tabular}


Nanoimpact Transformed Sample Oscillation Data: Distribution of Results

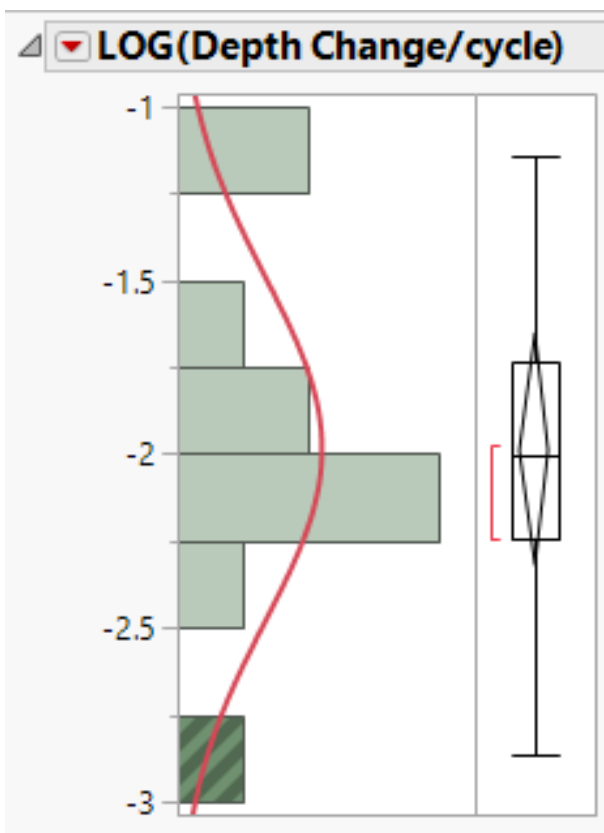

- Normal(-1.9853,0.49545)

\begin{tabular}{|c|c|c|}
\hline \multicolumn{3}{|c|}{$\triangle$ Quantiles } \\
\hline $100.0 \%$ & maximum & -1.146228144 \\
\hline $99.5 \%$ & & -1.146228144 \\
\hline $97.5 \%$ & & -1.146228144 \\
\hline $90.0 \%$ & & -1.163296998 \\
\hline $75.0 \%$ & quartile & -1.732547611 \\
\hline $50.0 \%$ & median & -2.004952687 \\
\hline $25.0 \%$ & quartile & -2.245889752 \\
\hline $10.0 \%$ & & -2.788344938 \\
\hline $2.5 \%$ & & -2.864704011 \\
\hline $0.5 \%$ & & -2.864704011 \\
\hline $0.0 \%$ & minimum & -2.864704011 \\
\hline
\end{tabular}




\begin{tabular}{|c|c|c|c|c|}
\hline \multicolumn{2}{|c|}{$\Delta \nabla$ Summary Statistics } & & & \\
\hline \multicolumn{2}{|c|}{ Mean -1085249} & \multicolumn{3}{|c|}{-1.985349} \\
\hline Std Dev & \multicolumn{4}{|c|}{0.4954452} \\
\hline Std Err Mean & \multicolumn{4}{|c|}{0.1493823} \\
\hline Upper $95 \%$ Mean & \multicolumn{4}{|c|}{-1.652505} \\
\hline Lower $95 \%$ Mean & \multicolumn{4}{|c|}{-2.318194} \\
\hline $\mathrm{N}$ & & 11 & & \\
\hline \multicolumn{5}{|c|}{$\Delta \nabla$ Fitted Normal } \\
\hline \multicolumn{5}{|c|}{$\triangle$ Parameter Estimates } \\
\hline Type & Parameter & Estimate & Lower $95 \%$ & Upper $95 \%$ \\
\hline Location $\mu$ & & -1.985349 & -2.318194 & -1.652505 \\
\hline Dispersion $\sigma$ & & 0.4954452 & 0.346176 & 0.8694734 \\
\hline \multicolumn{5}{|c|}{$-2 \log ($ Likelihood $)=14.7660789256195$} \\
\hline \multicolumn{5}{|c|}{$\Delta$ Goodness-of-Fit Test } \\
\hline \multicolumn{5}{|c|}{ Shapiro-Wilk W Test } \\
\hline W & \multicolumn{4}{|c|}{ Prob $<W$} \\
\hline 0.941772 & \multicolumn{4}{|c|}{0.5415} \\
\hline \multicolumn{5}{|c|}{$\begin{array}{l}\text { Note: } \mathrm{Ho}=\text { The data is from the Normal distribution. Small p- } \\
\text { values reject Ho. }\end{array}$} \\
\hline
\end{tabular}




\section{Nanoimpact Pendulum Oscillation Data: Distribution of Results}

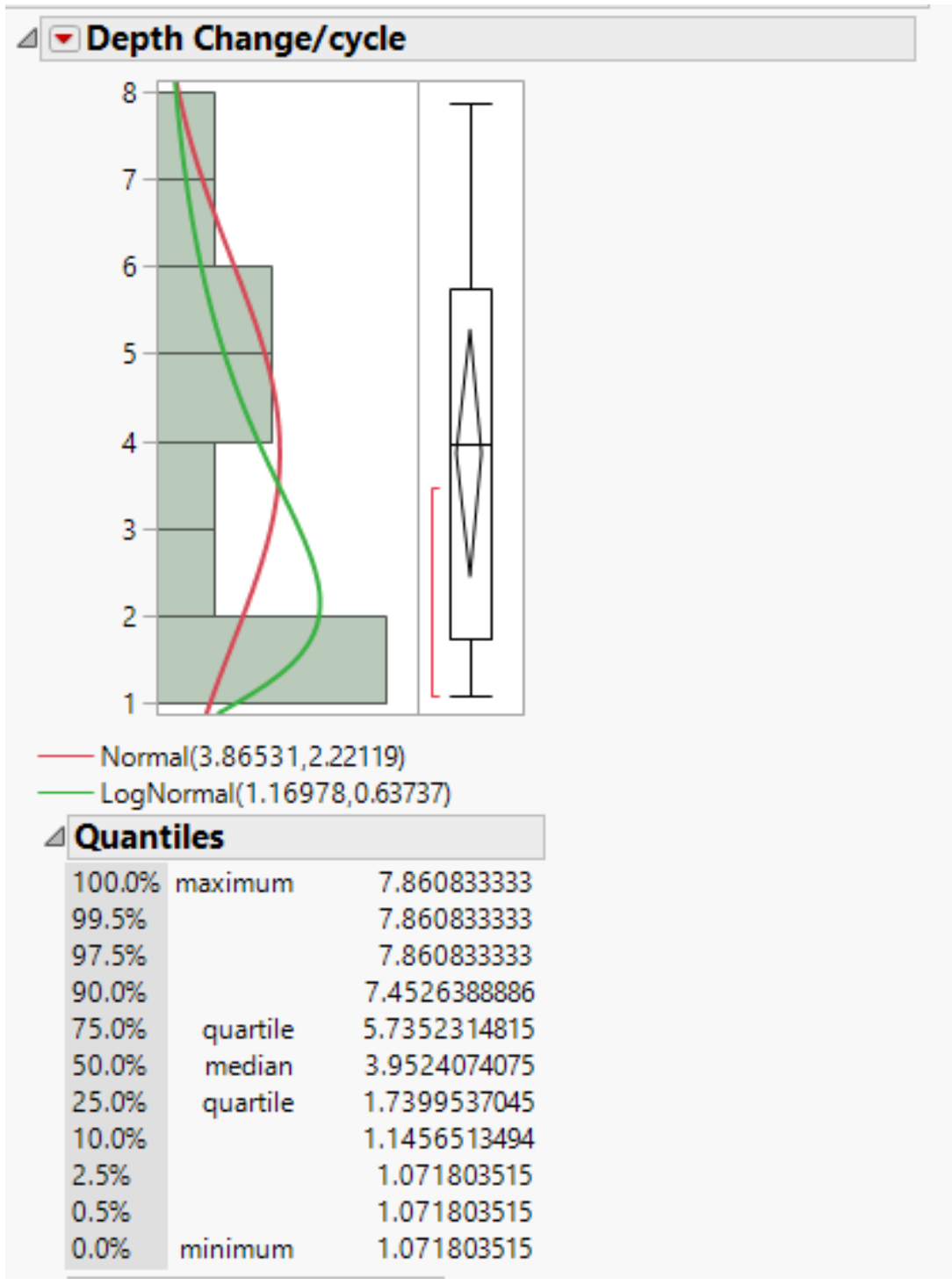




\begin{tabular}{|c|c|}
\hline \multicolumn{2}{|c|}{$\triangle \nabla$ Summary Statistics } \\
\hline Mean & 3.8653123 \\
\hline Std Der & \\
\hline Std Err Mean & 0.6412023 \\
\hline Upper $95 \%$ Mean & 5.2765891 \\
\hline Lower $95 \%$ Mean & 2.4540356 \\
\hline $\mathrm{N}$ & 12 \\
\hline
\end{tabular}

\section{$\Delta-$ Fitted LogNormal}

\section{$\triangle$ Parameter Estimates}

Type Parameter Estimate Lower 95\% Upper 95\%

\begin{tabular}{l|lll} 
Scale $\mu$ & 1.1697801 & 0.7782798 & 1.5612804
\end{tabular}

$\begin{array}{llll}\text { Shape } \sigma & 0.6373698 & 0.4477469 & 1.0109856\end{array}$

$-2 \log ($ Likelihood $)=51.3195206046943$

\section{$\triangle$ Goodness-of-Fit Test}

Kolmogorov's D

D Prob $>$ D

$0.193704>0.1500$

Note: $\mathrm{Ho}=$ The data is from the LogNormal distribution. Small pvalues reject $\mathrm{Ho}$. 
Nanoimpact Transformed Pendulum Oscillation Data: Distribution of Results

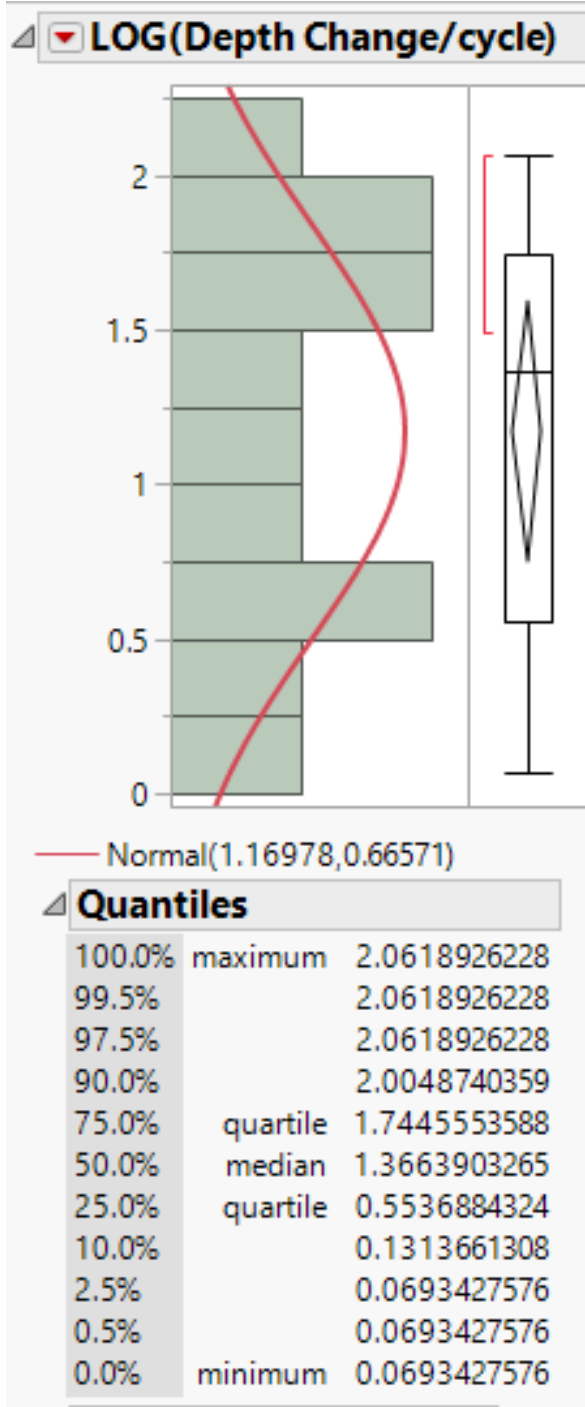




\begin{tabular}{|c|c|}
\hline \multicolumn{2}{|c|}{ - Summary Statistics } \\
\hline Mean & 1.1697801 \\
\hline Std Dev & 0.665711 \\
\hline Std Err Mean & 0.1921742 \\
\hline Upper $95 \%$ Mean & 1.5927527 \\
\hline Lower $95 \%$ Mean & 0.7468075 \\
\hline $\mathrm{N}$ & \\
\hline
\end{tabular}

$\triangle$ Fitted Normal

$\triangle$ Parameter Estimates Type Parameter Estimate Lower 95\% Upper 95\%

\begin{tabular}{ll|llll} 
Location $\mu$ & 1.1697801 & 0.7468075 & 1.5927527
\end{tabular}

\begin{tabular}{l|lll} 
Dispersion $\sigma$ & 0.665711 & 0.4715865 & 1.1302961
\end{tabular}

$-2 \log ($ Likelihood $)=23.2889339059494$

\section{$\triangle$ Goodness-of-Fit Test}

Shapiro-Wilk W Test

W Prob $<$ W

$0.934093 \quad 0.4255$

Note: $\mathrm{Ho}=$ The data is from the Normal distribution. Small pvalues reject $\mathrm{Ho}$. 


\section{Nanoimpact Data: Full ANOVA Results}

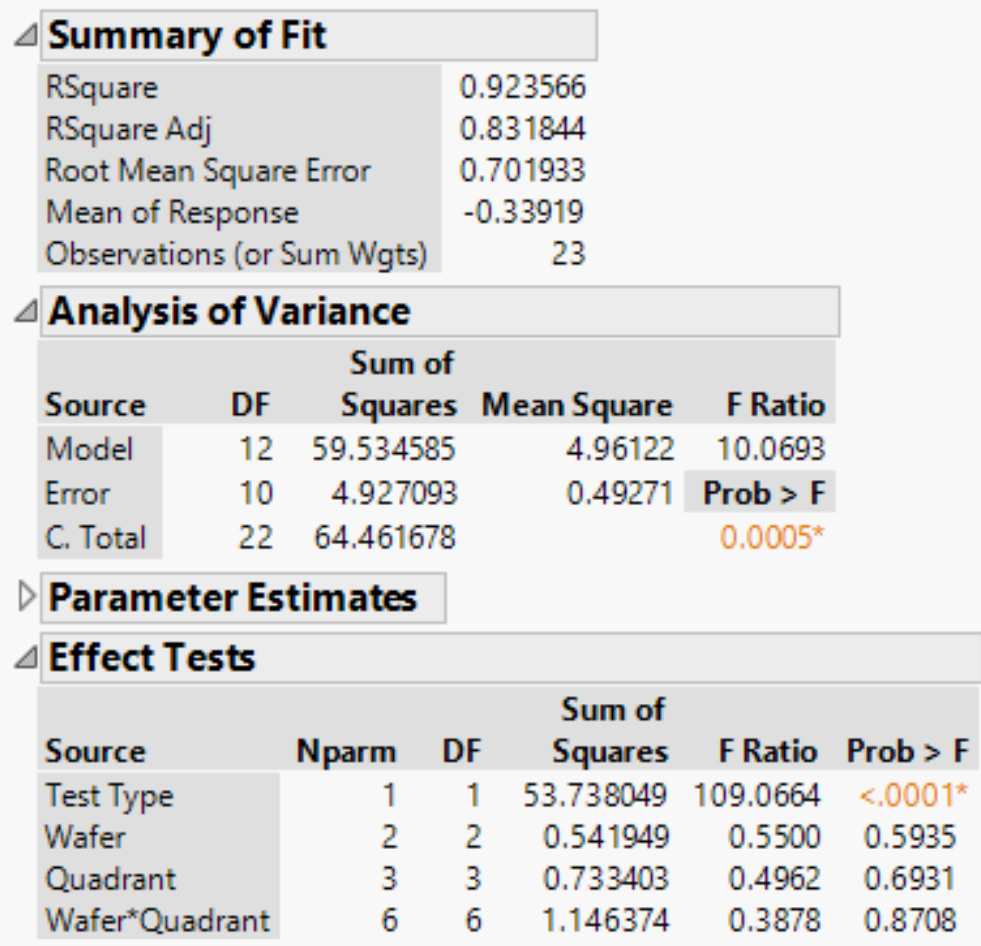

\section{$\triangle$ Residual by Predicted Plot}

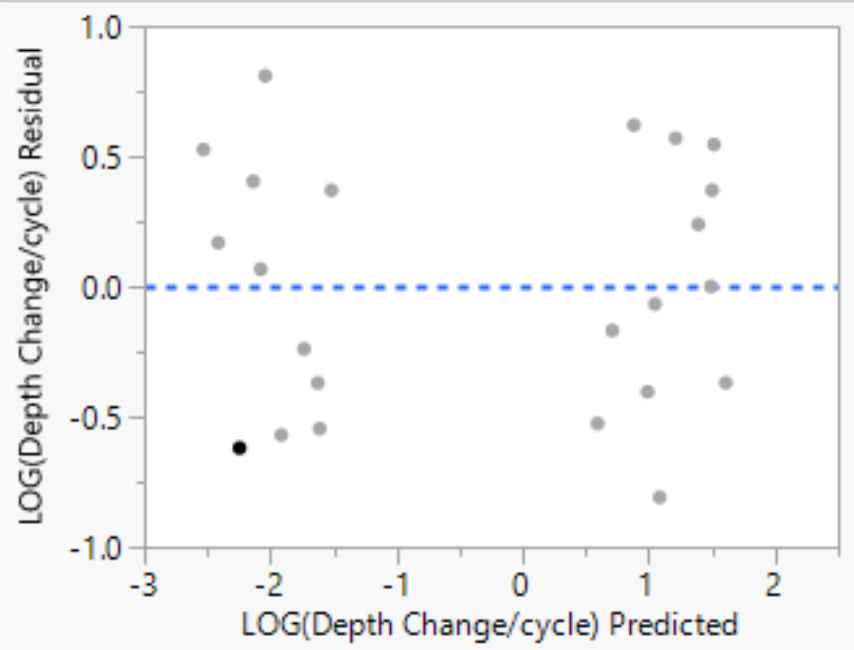




\section{Nanoscratch Data: Distribution of Results}

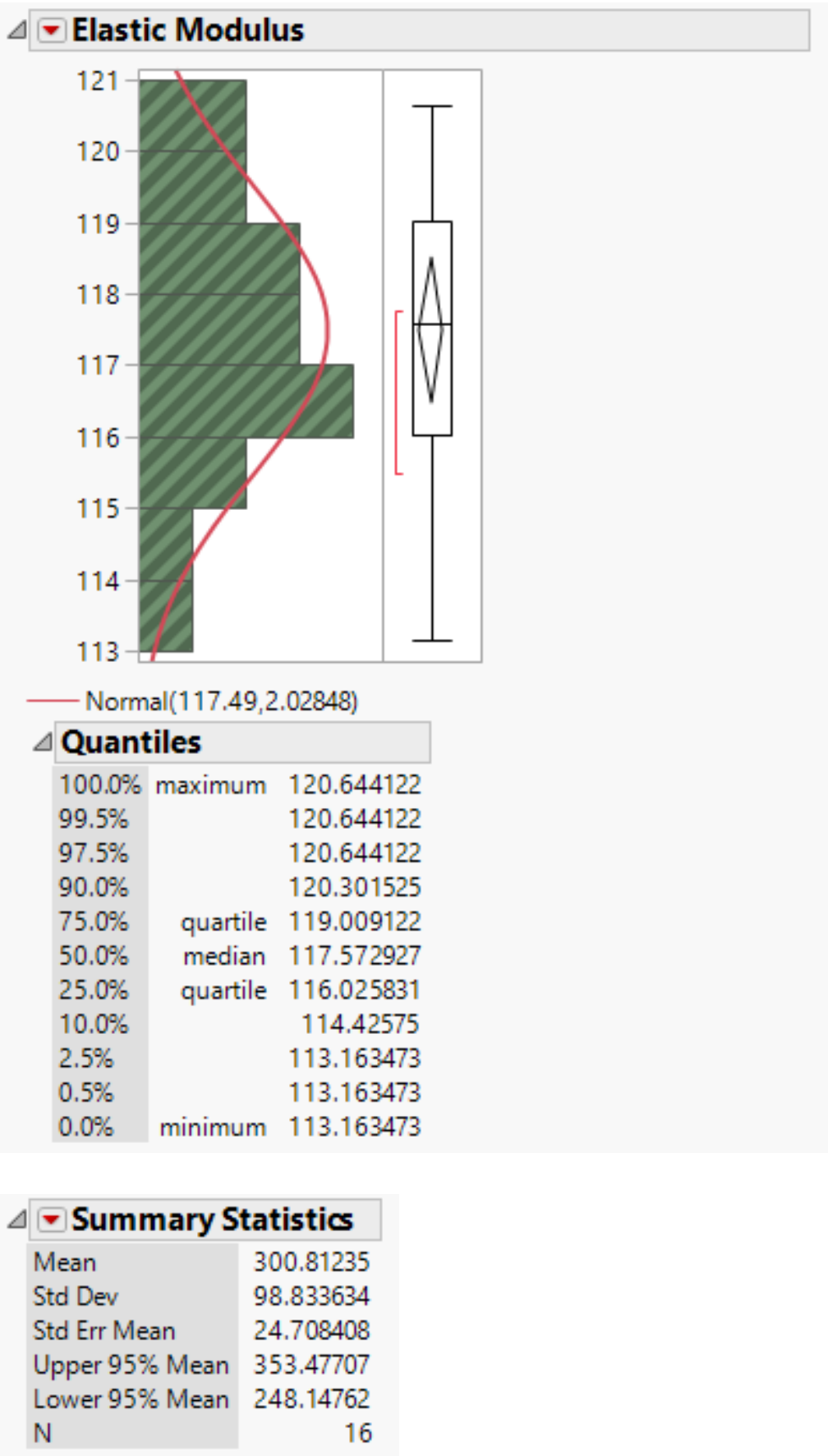




\begin{tabular}{|c|c|c|c|c|}
\hline \multicolumn{5}{|c|}{$\Delta \nabla$ Fitted Normal } \\
\hline \multicolumn{5}{|c|}{$\triangle$ Parameter Estimates } \\
\hline Type & Parameter & Estimate & Lower $95 \%$ & Upper $95 \%$ \\
\hline Location & $\mu$ & 300.81235 & 248.14762 & 353.47707 \\
\hline Dispersion & $\sigma$ & 98.833634 & 73.008885 & 152.96395 \\
\hline \multicolumn{5}{|c|}{$-2 \log ($ Likelihood $)=191.39604806269$} \\
\hline \multicolumn{5}{|c|}{$\Delta$ Goodness-of-Fit Test } \\
\hline \multicolumn{5}{|c|}{ Shapiro-Wilk W Test } \\
\hline W & \multicolumn{4}{|c|}{ Prob $<W$} \\
\hline 0.958650 & \multicolumn{4}{|c|}{0.6374} \\
\hline
\end{tabular}


Nanoscratch Data: Full ANOVA Results

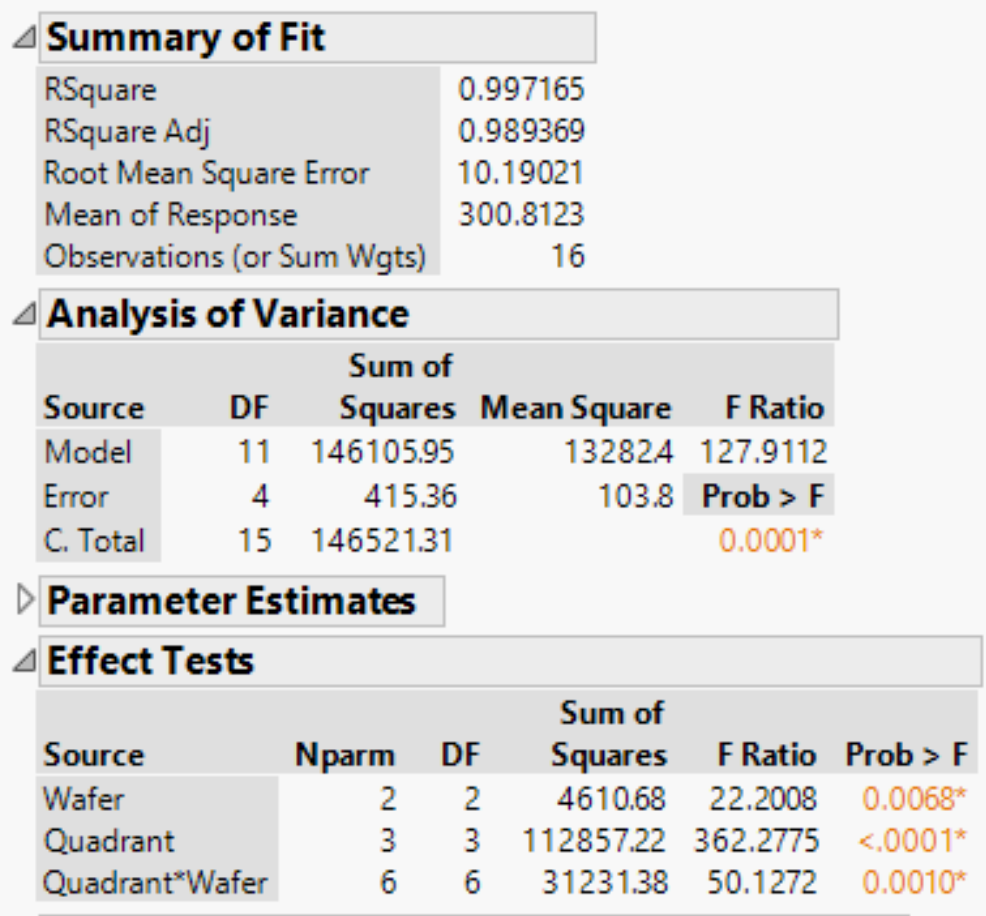

